\title{
Un modèle de développement et de déploiement de compétences pour les enseignants et les formateurs cliniques en sciences infirmières / A model of competency development and deployment for nurse academics and clinical educators
}

\author{
Martin Charette \\ Université de Montréal, m.charette@umontreal.ca \\ Johanne Goudreau \\ University of Montreal, johanne.goudreau@umontreal.ca \\ Anne Bourbonnais \\ Université de Montréal, anne.bourbonnais@umontreal.ca
}

Follow this and additional works at: https://qane-afi.casn.ca/journal

Part of the Other Nursing Commons

\section{Recommended Citation}

Charette, Martin; Goudreau, Johanne; and Bourbonnais, Anne (2019) "Un modèle de développement et de déploiement de compétences pour les enseignants et les formateurs cliniques en sciences infirmières / A model of competency development and deployment for nurse academics and clinical educators," Quality Advancement in Nursing Education - Avancées en formation infirmière: Vol. 5: Iss. 2, Article 6.

DOI: https://doi.org/10.17483/2368-6669.1188

This Article is brought to you for free and open access by Quality Advancement in Nursing Education - Avancées en formation infirmière. It has been accepted for inclusion in Quality Advancement in Nursing Education - Avancées en formation infirmière by an authorized editor of Quality Advancement in Nursing Education - Avancées en formation infirmière. 


\section{Un modèle de développement et de déploiement de compétences pour les enseignants et les formateurs cliniques en sciences infirmières / A model of competency development and deployment for nurse academics and clinical educators}

\section{Cover Page Footnote}

Financement: Ce projet a été financé par les Instituts de recherche en santé du Canada (IRSC), le Ministère de l'Enseignement supérieur, de la Recherche et du Sport (programme MESRS-Université), à la Faculté des sciences infirmières et à la Faculté des études supérieures et postdoctorales de l'Université de Montréal.

\section{Erratum}

- Référence aux pages 14 et 29 a été modifiée de "Charette, M., Goudreau, J., \& Bourbonnais, A. (2019b, early view). Organizational and individual factors influencing the practice of new graduate nurses: a focused ethnography of acute care settings. Journal of Clinical Nursing. https://doi.org/10.1111/ jocn.14959" à "Charette, M., Goudreau, J., \& Bourbonnais, A. (2019b). Factors influencing the practice of new graduate nurses: a focused ethnography of acute care settings, Journal of Clinical Nursing, 28(19/ 20), 3618-3631. https://doi.org/10.1111/jocn.14959" - Référence aux pages 14 et 29 a été modifiée "Ke, Y.-T., Kuo, C.-C., et Hung, C.-H. (2017). The effects of nursing preceptorship on new nurses' competence, professional socialization, job satisfaction and retention: A systematic review. Journal of Advanced Nursing, 2017, 1-10. https://doi.org/10.1111/jan.13317" à "Ke, Y. T., Kuo, C. C., \& Hung, C. H. (2017). The effects of nursing preceptorship on new nurses' competence, professional socialization, job satisfaction and retention: A systematic review. Journal of Advanced Nursing, 73(10), 2296-2305. https://doi.org/ 10.1111/jan.13317" - Reference on pages 14 and 29 changed from "Charette, M., Goudreau, J., \& Bourbonnais, A. (2019b, early view). Organizational and individual factors influencing the practice of new graduate nurses: a focused ethnography of acute care settings. Journal of Clinical Nursing. https://doi.org/10.1111/jocn.14959" to "Charette, M., Goudreau, J., \& Bourbonnais, A. (2019b). Factors influencing the practice of new graduate nurses: a focused ethnography of acute care settings, Journal of Clinical Nursing, 28(19/20), 3618-3631. https://doi.org/10.1111/jocn.14959" - Reference on page 15 changed from "Ke, Y.-T., Kuo, C.-C., et Hung, C.-H. (2017). The effects of nursing preceptorship on new nurses' competence, professional socialization, job satisfaction and retention: A systematic review. Journal of Advanced Nursing, 2017, 1-10. https://doi.org/10.1111/jan.13317" to "Ke, Y. T., Kuo, C. C., \& Hung, C. H. (2017). The effects of nursing preceptorship on new nurses' competence, professional socialization, job satisfaction and retention: A systematic review. Journal of Advanced Nursing, 73(10), 2296-2305. https://doi.org/10.1111/jan.13317" - Terminology change: "implementation" changed to "deployment" in English content 


\section{Introduction}

Les établissements d'enseignement offrant des programmes de formation initiale en sciences infirmières réforment constamment ces programmes afin d'offrir un enseignement de qualité et de s'arrimer avec les besoins de la population. Malheureusement, la communication est souvent déficiente entre les mondes académique et clinique (Saifan, AbuRuz, et Masa'deh, 2015), créant une confusion et un sentiment de manque de préparation chez les infirmiers nouvellement diplômés qui sont en transition d'étudiant à professionnel (Duchscher, 2009). De plus, les enseignants dans les établissements d'enseignement et les formateurs dans les milieux cliniques ne partagent pas toujours la même vision du concept de compétence (Blanchet Garneau, Lavoie, et Grondin, 2017), ajoutant une certaine confusion à une situation déjà problématique. Dadgaran, Parvizy, et Peyrovi (2012) suggèrent que la transformation des programmes de formation initiale vers une approche misant sur une formation pratique avancée (incluant des laboratoires, de la simulation clinique haute-fidélité et des stages en milieux cliniques) et mettant l'accent sur l'interdépendance entre la pratique et les connaissances théoriques pourrait aider à améliorer la communication entre les mondes académique et clinique.

Une telle approche pourrait être l'approche par compétences (APC). L'Institute of Medicine (IOM) concluait en 2003 qu'une transformation des programmes de formation des professionnels de la santé basée sur l'APC permettrait de mieux préparer ces derniers aux défis des systèmes de santé de demain et de travailler en collaboration les uns avec les autres. Ceci pourrait pallier les lacunes majeures identifiées par l'IOM dans la qualité des soins offerts aux patients, tributaires d'une formation initiale inadéquate des professionnels de la santé.

L'APC, telle que promue par l'IOM, oblige à considérer l'apprentissage comme un processus actif et contextualisé, préparant les étudiants à agir en situation réelle. L'utilisation de méthodes d'enseignement et d'apprentissage (MEA) actives, telles que l'apprentissage par problèmes ou la simulation clinique, témoignent du passage du paradigme de l'enseignement vers le paradigme de l'apprentissage, soit d'une pédagogie passive centrée sur la transmission des savoirs de l'enseignant aux étudiants vers une approche active où l'étudiant est le principal acteur de ses apprentissages (Tardif, 2006). Dans la francophonie, une définition d'une compétence souvent reprise est celle de Tardif (2006, p. 22), soit "un savoir-agir complexe prenant appui sur la mobilisation et la combinaison efficaces d'une variété de ressources internes et externes à l'intérieur d'une famille de situations. » Cette définition est cohérente avec la vision holistique d'une compétence à laquelle adhèrent plusieurs auteurs en sciences de 1'éducation (Gonczi, 1996; Gonczi et Hager, 2010; Lasnier, 2000; Poumay et Georges, 2017) et en sciences infirmières (Blanchet Garneau et al., 2017; Charette, Goudreau, et Alderson, 2014; Garside et Nhemachena, 2013; Smith, 2012).

L'APC invite à placer l'étudiant au cœur de son processus d'apprentissage, entre autres par l'action (Tardif, 2006). De plus, certains auteurs spécifient que la réflexion dans l'action et sur l'action est tout aussi importante que l'action elle-même pour l'apprentissage (Pastré, 1999; Perrenoud, 2004; Schön, 1993) et que le développement d'habiletés réflexives augmente la perception de cohérence entre les savoirs théoriques et les habiletés pratiques (Hatlevik, 2012). Des activités de pratique réflexive peuvent autant être implantées autant dans un programme de formation initiale auprès d'étudiants en sciences infirmières que dans le cadre d'une activité de développement professionnel continu auprès d'infirmiers.

Alors que l'APC est souvent adoptée par des établissements d'enseignement pour leurs programmes de formation initiale, certains établissements de santé emboitent le pas et mettent sur pied des programmes de transition de nouveaux diplômés ou de développement 
professionnel également basés sur l'APC. L'utilisation d'un langage commun partagé par les enseignants et les formateurs cliniques, en particulier en ce qui concerne les notions de développement et de déploiement de compétences, permettrait de situer les attentes envers les étudiants et les nouveaux diplômés sur un continuum débutant lors de la formation initiale et se poursuivant dans le milieu clinique. La collaboration entre les milieux académiques et cliniques devient alors un élément facilitant le transfert des connaissances et l'intégration des apprentissages, favorisant une pratique infirmière de qualité. Un modèle théorique liant des théories de l'apprentissage à la pratique infirmière en milieu clinique pourrait potentiellement offrir un tel langage commun. Cet article propose un modèle de développement et de déploiement de compétences en pratique infirmière.

\section{Fondements théoriques}

Ce modèle a été construit théoriquement par l'intégration des travaux de Benner sur le développement de l'expertise professionnelle à l'APC. Avant de présenter le modèle en question, ses fondements théoriques seront décrits, soit l'APC, en portant une attention au concept de compétence, ainsi que les écrits de Benner sur le développement de l'expertise professionnelle, son modèle de novice à expert ainsi que sa vision pédagogique seront présentés.

\section{Approche par compétences}

Depuis un siècle, trois réformes majeures ont transformé les programmes de formation en sciences de la santé. La première grande réforme a suivi la publication des rapports Flexner (1910), Goldmark (1923) et Weir (1932), menant à la création de programmes de médecine et de sciences infirmières basés sur l'acquisition de connaissances générées par les sciences biomédicales. Bien avant ces rapports, Florence Nightingale avait mis en place la première école infirmière indépendante des hôpitaux au milieu du XIX ${ }^{\mathrm{e}}$ siècle (Pepin, Ducharme, et Kérouac, 2017). Première infirmière chercheuse, Nightingale croyait en la formation scientifique des infirmiers. Dans son rapport de 1932, Weir argumentait qu'il fallait considérer les soins infirmiers comme une profession en devenir, plutôt que sous l'hégémonie de la médecine. Les connaissances enseignées aux infirmiers devraient donc être adaptées à leur contexte professionnel propre.

À partir des années 1950, une idée prend de l'ampleur dans les milieux de l'enseignement : la transmission de savoirs par des experts n'est plus suffisante pour former des professionnels compétents (Guillemette et Gauthier, 2008). Cette réflexion a mené à une seconde réforme majeure, dans laquelle l'utilisation adéquate des savoirs devenait aussi, sinon plus, importante que l'acquisition du savoir même. Cette réforme a surtout été marquée par l'introduction de MEA actives comme l'apprentissage par problèmes dans certains programmes de formation de professionnels de la santé (Frenk et al., 2010). Le terme compétence a alors pris une connotation de performance en situation et ces programmes ont été étiquetés comme étant basés sur une APC de première génération. Afin de guider ces programmes, des référentiels de compétences ont été élaborés, généralement par une analyse béhavioriste du travail du professionnel, axé sur la réalisation de tâches en situation simple ou complexe, donnant lieu à des référentiels de centaines, voire des milliers, de compétences dans certains cas (Ishler, 1974).

Cette première génération d'APC a été critiqué, puisqu'elle semblait prioriser la performance plutôt que les processus internes cognitifs liés à l'action du professionnel et son apprentissage. Cet accent sur des comportements précis a amené certains auteurs à la considérer comme une APC béhavioriste (Guillemette et Gauthier, 2008). En réaction à cette $\mathrm{APC}$ de première génération, des réflexions ont mené vers une APC de deuxième génération 
prenant appui sur le cognitivisme et le constructivisme (Gonczi et Hager, 2010; Goudreau et al., 2009). Alors que la première génération de l'APC a mis en lumière l'importance de l'action et de la performance dans les comportements des professionnels (Guillemette et Gauthier, 2008), la seconde génération invite à considérer la compétence comme l'intégration de l'action et des processus cognitifs qui la sous-tendent, à travers la complexité singulière des situations vécues par le professionnel (Tardif, 2006).

Le cognitivisme, courant issu de la psychologie moderne, s'oriente autour de l'acquisition et du traitement de l'information, en faisant transiger par la mémoire à court terme, aussi appelée mémoire de travail, et en échangeant les informations entre celle-ci et la mémoire à long terme (Billings et Halstead, 2016, Tardif, 2016). Les différents types de connaissances dont les étudiants s'approprient (connaissances déclaratives, procédurales et conditionnelles; Boulet, Savoie-Zajc, et Chevrier, 1996) peuvent par la suite être mobilisés et combinés, leur permettant d'agir dans une variété de situations (Tardif, 2006). Le constructivisme, second courant à la base de l'APC de deuxième génération (Billings et Halstead, 2016), concerne l'intégration de nouvelles connaissances qui se fait à partir des connaissances antérieures, par la construction de schèmes cognitifs complexes (Tardif, 2016), qui donneront à l'apprenant un répertoire d'actions de plus en plus grand et la transférabilité de ses apprentissages dans diverses situations (Steiner, 2001). Les relations sociales contribuent de façon importante à la construction des savoirs, ce que certains appellent le socioconstructivisme (Tardif, 2016). Par des échanges avec ses pairs, les schèmes cognitifs de l'apprenant sont ébranlés et enrichis par une déconstruction et une reconstruction de ces schèmes. Certains apprentissages ne peuvent se faire en vase clos et arrivent uniquement lorsque l'apprenant est aidé ou appuyé par une tierce personne; ce concept est appelé une zone proximale de développement (Shabani, Khatib et Ebadi, 2010). L'enseignant et les autres étudiants ont donc un rôle primordial dans l'apprentissage d'une personne; l'enseignant doit tenter de varier les MEA et la formule de ces activités afin de favoriser les échanges entre les individus.

Considérant ces deux courants fondamentaux (trois si l'on considère le socioconstructivisme comme un courant propre), l'APC de deuxième génération place les étudiants au cœur de leurs apprentissages, grâce à des activités s'appuyant sur une variété de MEA actives. L'enseignant tente de favoriser une compréhension approfondie des connaissances, une intégration de différents types de connaissances et un transfert des apprentissages dans de multiples situations complexes et variées, plutôt que de viser la mémorisation d'une grande quantité de connaissances (Tardif, 2006). En sciences de la santé, la visée de ces programmes basés sur l'APC est de préparer les futurs professionnels à la réalité des systèmes de santé d'aujourd'hui (Frenk et al., 2010). Le développement de compétences communes, mais adaptées au contexte professionnel de chacun, pourrait favoriser une collaboration interprofessionnelle répondant aux besoins de santé de la population.

À travers la littérature en sciences de l'éducation, plusieurs définitions du concept de compétence peuvent être classés selon une vision béhavioriste, cohérente avec l'APC de première génération, ou selon une vision holistique, davantage cohérente avec l'APC de deuxième génération. La définition comportementaliste fait référence à la notion de performance; cette vision provient de l'industrialisation, où une personne pouvait être jugée compétente simplement à partir des actions qu'elle effectuait (Tardif, 2006). La vision holistique, quant à elle, englobe une variété de savoirs (savoir, savoir-faire, savoir-être) qu'une personne possède et qui lui permet d'être compétente, en plus d'identifier que les compétences d'une personne sont intimement liées au contexte dans lequel elle évolue 
(Gonczi et Hager, 2010). La définition de Tardif (2006) citée précédemment entre dans cette catégorie.

Du côté des sciences infirmières, une analyse critique de la littérature sur le concept de compétence en formation infirmière a été effectuée par Charette et al., 2014. Cette analyse a permis de constater qu'à l'instar des auteurs en sciences de l'éducation, la majorité des auteurs en sciences infirmières utilisent une définition holistique du concept de compétences, cohérente avec l'APC de deuxième génération. L'analyse de 41 articles publiés entre 2008 et 2013 a permis d'identifier des attributs et des conséquences du concept de compétence. Les attributs identifiés sont les caractéristiques essentielles qui ont permis de redéfinir le concept de compétence à titre d'une "mise en action d'habiletés systémiques, contextualisée et qui est en continuel développement» (Charette et al., 2014, p. 37). Dans cette définition, l'attribut «habiletés systémiques » représente une symbiose d'habiletés psychomotrices, cognitives et affectives, rappelant la notion de savoir-agir complexe de Tardif (2006) ou de savoirs, habiletés et attitudes (Cronenwett et al., 2007). Les conséquences du concept qui ont été dégagées correspondent à ce qui peut survenir lorsque le concept est mis en œuvre; Charette et al. (2014) ont identifié que la protection du public, la satisfaction professionnelle et le dialogue entre praticiens sont des conséquences qui surviennent lorsque les infirmiers agissent avec compétences.

Finalement, plusieurs auteurs abordent le concept d'évaluation de compétences en formation infirmière (Garside et Nhemachena, 2013; Meretoja et Koponen, 2012; Rischel, Larsen, et Jackson, 2008). Ces auteurs précisent que pour être cohérent avec une définition holistique du concept de compétences, les infirmiers ne devraient pas être jugés compétents uniquement à partir de leurs habiletés techniques (p. ex., l'évaluation de la rapidité d'exécution d'une tâche), qui ne représentent qu'un aspect de la pratique infirmière. Comme le concept de compétence est complexe et composé de multiples facettes, l'évaluation devrait refléter cette complexité (Franklin et Melville, 2015). Ces auteurs, tout comme Tardif (2006) d'ailleurs, préconisent que l'évaluation soit effectuée à plusieurs moments afin de rendre compte d'une trajectoire de développement de l'apprenant plutôt que d'un cliché instantané.

En résumé, l'APC de seconde génération, basée sur le cognitivisme et le constructivisme, permet de considérer l'apprentissage comme un processus dans lequel l'étudiant est actif. Diverses MEA seront réfléchis par l'enseignant pour aider l'étudiant à acquérir différents types de connaissances qui lui permettront d'agir dans des situations professionnelles authentiques et de développer son expertise professionnelle.

\section{Expertise professionnelle : de novice à expert}

Patricia Benner a étudié le phénomène du développement de l'expertise professionnelle en adaptant le modèle d'acquisition d'habiletés de Dreyfus et Dreyfus (1980) aux sciences infirmières, ces derniers l'ayant développé en étudiant les habiletés des pilotes d'avion de chasse de l'armée de l'air des États-Unis. À la suite d'une étude multicentrique auprès de 62 infirmiers et 5 étudiants en sciences infirmières, Benner a conclu au début des années 1980 que les cinq stades du modèle des frères Dreyfus étaient transférables en sciences infirmières et elle les a adaptés à ce contexte (voir Tableau 1; Benner, 1984). Elle a caractérisé chaque stade en spécifiant que le changement d'un stade à l'autre ne s'effectue pas de façon automatique, mais survient uniquement si l'infirmier s'engage dans un processus continu d'apprentissage expérientiel. Bien que le stade expert soit le dernier stade du modèle, il ne représente pas une finalité. Il s'agit plutôt d'une période où l'infirmier poursuit son processus de développement en innovant sa pratique tout en restant engagé dans son apprentissage. 
Tableau 1

Brève description des stades du modèle de novice à expert (inspiré de Benner, 1984)

\begin{tabular}{|c|l|}
\hline Stade & \multicolumn{1}{c|}{ Description brève } \\
\hline $\begin{array}{c}\text { Novice } \\
\text { (novice) }\end{array}$ & $\begin{array}{l}\text { Aucune expérience clinique. Le novice se base principalement sur des } \\
\text { notions théoriques et des règles strictes afin d'arriver à des conclusions et } \\
\text { guider ses comportements. }\end{array}$ \\
\hline $\begin{array}{c}\text { Débutant } \\
\text { advanced } \\
\text { beginner })\end{array}$ & $\begin{array}{l}\text { L'apprenant débutant a des difficultés d'adaptation au nouveau milieu et se } \\
\text { sent dépassé et incompétent. Il ne parvient pas toujours à bien s'organiser ou } \\
\text { prioriser ses interventions, mais démontre un niveau acceptable de pratique. }\end{array}$ \\
\hline $\begin{array}{c}\text { Compétent } \\
\text { competent })\end{array}$ & $\begin{array}{l}\text { L'infirmier organise mieux ses soins en se basant sur ses expériences } \\
\text { antérieures. Il parvient à planifier ses interventions à moyen et long terme et } \\
\text { est capable de réagir en situation complexe. Il distingue certains éléments les } \\
\text { plus importants d'une situation. }\end{array}$ \\
\hline $\begin{array}{c}\text { Performant } \\
\text { proficient })\end{array}$ & $\begin{array}{l}\text { L'infirmier perçoit la situation de façon holistique. Il distingue les éléments } \\
\text { saillants et capte les changements subtils. Cette discrimination se fait parfois } \\
\text { inconsciemment (intuition). Ceci lui permet d'intervenir de façon appropriée } \\
\text { et prompte. }\end{array}$ \\
\hline $\begin{array}{c}\text { Expert } \\
\text { (expert) }\end{array}$ & $\begin{array}{l}\text { L'infirmier a une vision à plus long terme des interventions nécessaires pour } \\
\text { chaque patient. Grâce à son bagage d'expériences cliniques, il saisit de façon } \\
\text { intuitive ce qui doit être fait dans chaque situation. Cette sagesse pratique lui } \\
\text { permet d'innover sa pratique lors de situations complexes. }\end{array}$ \\
\hline
\end{tabular}

À travers les années, les travaux de Benner sur le développement de l'expertise professionnelle ont été étiquetés de plusieurs façons : modèle de novice à expert, stades de compétence clinique, théorie de novice à expert, théorie de l'acquisition d'habiletés infirmières. Cependant, d'un point de vue épistémologique, un modèle et une théorie ne sont pas équivalents, et il existe différents niveaux de théorie (p. ex., une théorie pratique, une théorie de niveau intermédiaire ou une théorie à large spectre; Risjord, 2010). Une des raisons pouvant expliquer cette confusion en ce qui a trait aux travaux de Benner provient d'un débat paradigmatique sur la validité de la recherche interprétative pour l'avancement des connaissances disciplinaires, débat qui était à son apogée dans les années 1970-80, mais qui semble perdurer encore à ce jour. Benner fût une des premières infirmières chercheuses à faire émerger des savoirs esthétiques incorporés dans la pratique en utilisant une méthode qualitative. Ce faisant, elle a permis d'établir l'utilité de ce paradigme pour la recherche infirmière (Risjord, 2010). Cependant, ses travaux sur le développement de l'expertise professionnelle ne rencontrent pas le niveau d'abstraction nécessaire pour être une théorie. Ils devraient plutôt être considéré comme un modèle théorique, puisqu'ils ont permis de décrire un phénomène et les stades associés, et ce, par des données empiriques (Risjord, 2010).

En plus de cette pluralité de titre pour désigner les travaux de Benner, une autre confusion règne sur les deux premiers stades du modèle de novice à expert, soit ceux de novice et de débutant. Certains interprètent qu'un apprenant au stade novice est un infirmier nouvellement diplômé et que le modèle ne s'intéresse donc pas à la formation initiale, alors que d'autres considèrent le novice comme étant un étudiant en formation. Or, dans un article de 1992 détaillant les résultats d'une étude qualitative pour approfondir le modèle, Benner, Tanner et Chesla précisent que le second stade, celui de débutant, correspond aux infirmiers nouvellement diplômés. Cette précision peut sembler banale, mais elle confirme que le 
développement de l'expertise professionnelle selon Benner débute par un programme de formation initiale et englobe la transition des infirmiers entre les milieux académique et clinique.

Explicitement, le modèle de novice à expert fait mention d'acquisition d'habiletés et non de développement de compétences. Cependant, Benner (1984) juge qu'il faut considérer ces habiletés dans le cadre d'une performance holistique, liée au contexte de pratique de l'infirmier et qu'il ne faut pas considérer l'action d'un infirmier uniquement d'un point de vue procédural. Ceci s'apparente à la notion de symbiose d'habiletés systémiques contextualisées de Charette et al. (2014) ou encore à la notion de savoir-agir complexe et contextualisé de Tardif (2006).

D'autres écrits plus récents de Benner mettent en lumière sa vision pédagogique pour la formation des infirmiers (Benner, Sutphen, Leonard, et Day, 2010; Benner, Tanner, et Chesla, 2009). Plus spécifiquement, elle milite pour une transformation des programmes de formation vers une pédagogie intégrative qui permet de faire un rapprochement entre les connaissances et l'expérience ou entre la théorie et la pratique (Benner et al., 2010). Ces réflexions de Benner sur la formation des infirmiers s'appuient sur les écrits de Dewey sur l'apprentissage expérientiel (Benner et al., 2009). Philosophe américain du courant du pragmatisme, Dewey (1997) a fait la promotion de l'éducation progressive, soit une pédagogie où l'expérience constitue le fondement de la connaissance et du moteur de l'apprentissage de l'étudiant, et où cette connaissance sert à générer l'action humaine (Morgan, 2014). Dans ce courant philosophique, l'accent est mis sur l'interaction entre l'apprenant et son environnement, social ou physique. Selon les situations rencontrées, les apprenants développent des prédispositions et des capacités à agir qui sont circonscrites dans un contexte précis, autant historique que culturel (Morgan, 2014). Les apprentissages d'une personne sont donc corollaires du contexte et des situations rencontrées, ce que certains auteurs appellent une cognition située ou contextualisée, rappelant le caractère contextuel des compétences (Tardif, 2006).

La vision pédagogique de Benner s'appuie sur ce concept de cognition située et d'expérience ancrée dans un contexte précis (Benner et al., 2009). Plus l'infirmier se trouve à un stade avancé du développement de son expertise, plus il est en mesure de saisir la globalité et l'unicité de chaque situation. Il sera donc en mesure d'intervenir en fonction de la situation, à en capter les éléments significatifs (ce que Benner appelle sense of salience) et à adapter sa pratique à cette unicité (Benner et al., 2009). Pour Benner, l'infirmier doit s'engager dans son apprentissage et dans une relation patient-infirmier profonde et significative, ce qui lui permettra d'en tirer des apprentissages. Ce savoir expérientiel doit toutefois être complété ou appuyé par des savoirs empiriques afin que l'infirmier puisse atteindre son plein potentiel; Benner insiste sur l'interdépendance de la recherche, de la théorie et de la pratique (Benner et al., 2009).

\section{Modèle de développement et déploiement de compétences en pratique infirmière}

De nombreux liens peuvent être faits entre les travaux de Benner sur le développement de l'expertise professionnelle et sa vision pédagogique, et l'APC de seconde génération. Par l'intégration de ces deux approches, un modèle de développement et déploiement de compétences en pratique infirmière a été construit (voir Figure 1) pour guider une étude qualitative qui avait pour but de décrire le déploiement de compétences d'infirmiers nouvellement diplômés (Charette, Goudreau et Bourbonnais, 2019a, 2019b). Les concepts-clés décrits par Benner, soit la contextualisation, l'apprentissage expérientiel, la réflexion sur la pratique et une progression à travers différents stades, sont également au cœur 
de l'APC, qui permet d'opérationnaliser ces concepts dans les programmes de formation initiale et de développement professionnel continu.

Ce modèle pourrait guider les enseignants et les formateurs cliniques dans l'élaboration d'activités de formation en identifiant des ingrédients essentiels : les MEA actives à prioriser, les compétences à développer et leur niveau (réel et attendu), le contexte de soins et la qualité des soins offerts aux patients. De plus, le positionnement de l'apprentissage expérientiel et de la réflexion au cœur du modèle est bien intentionnel, le développement de l'expertise reposant sur ces concepts-clés. Finalement, par l'illustration du continuum entre la formation et la pratique infirmière, ce modèle favorise l'utilisation d'un langage commun en termes d'apprentissages et de développement de compétences entre les milieux académique et clinique. Ce faisant, un plus fort sentiment de cohérence entre la formation initiale et le programme d'orientation dans lequel les infirmiers sont accueillis en début de carrière devrait être ressenti, permettant une transition plus aisée pour ces nouveaux infirmiers.

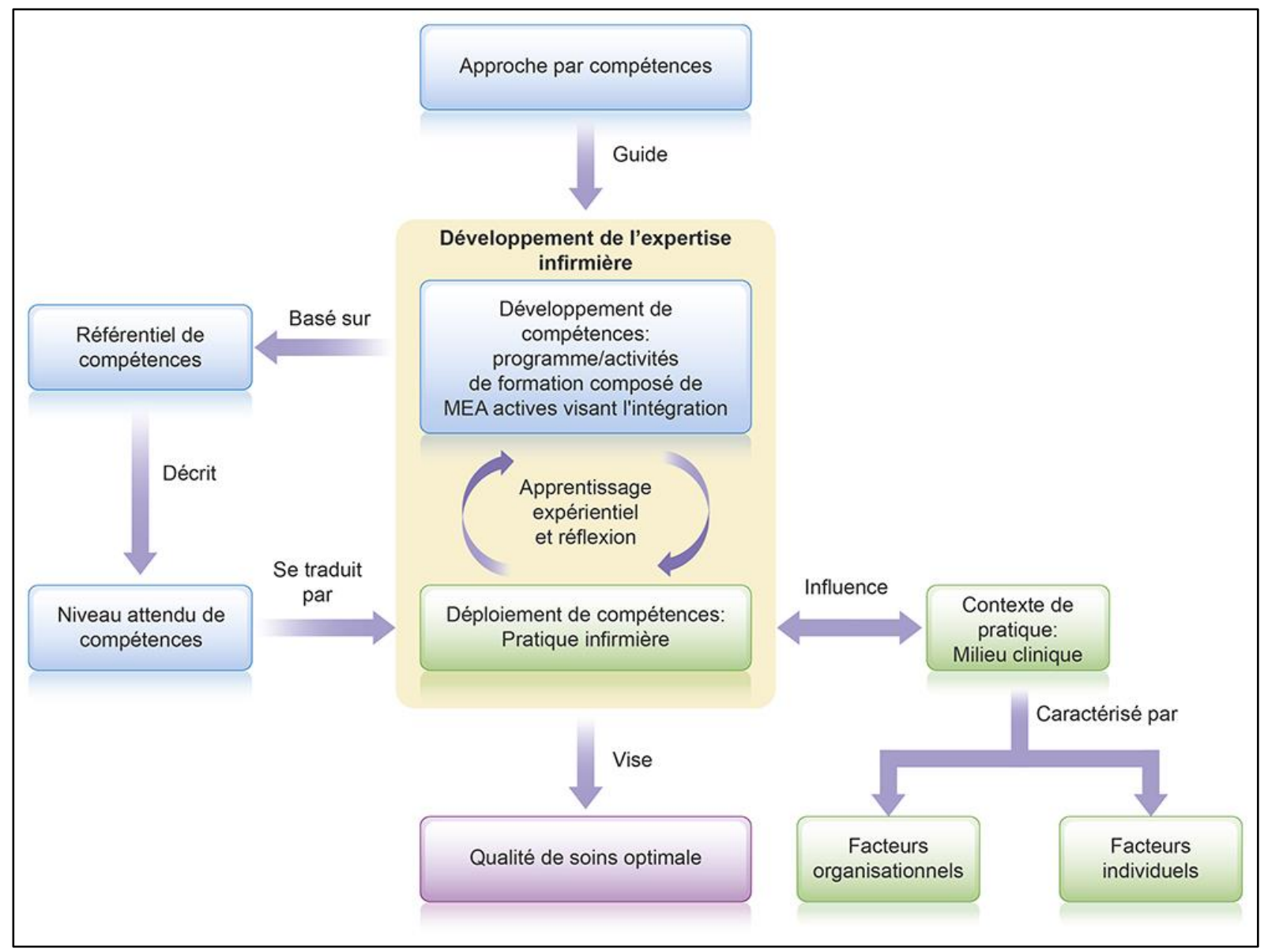

Figure 1 : Modèle de développement et déploiement de compétences en pratique infirmière

\section{Développement de l'expertise infirmière}

Au centre de ce modèle, on trouve le concept de développement de l'expertise infirmière décrit par Benner (Benner, 1984; Benner et al., 2009), qui englobe à la fois le développement et le déploiement de compétences. Le développement réfère au processus continu d'acquisition et d'intégration de ressources et connaissances diverses alors que déploiement correspond plutôt à la mise en action de cette symbiose d'habiletés en situation authentique. 
Afin de promouvoir le développement de compétences des étudiants (novices) et être cohérent avec l'APC de seconde génération, un programme ou une activité de formation devrait être constitué de MEA actives (Benner et al., 2010; Tardif, 2006), telles que l'apprentissage par problème, la simulation clinique haute-fidélité et les stages cliniques. Ces activités de formation doivent être planifiées en fonction de principes pédagogiques tels que ceux définis par Lasnier (2000) et brièvement expliqué au Tableau 2.

Tableau 2

Principes pédagogiques (inspiré de Lasnier, 2000, p. 158-174)

\begin{tabular}{|l|l|}
\hline \multicolumn{1}{|c|}{ Principe } & \multicolumn{1}{c|}{ Brève explication } \\
\hline Globalité & $\begin{array}{l}\text { Utilisation de situations globales et complexes afin de donner une vision } \\
\text { d'ensemble de la situation d'apprentissage }\end{array}$ \\
\hline Construction & $\begin{array}{l}\text { Activation des acquis antérieurs, élaboration de liens entre les ces acquis et } \\
\text { les nouveaux apprentissages et organisation de l'information en schème }\end{array}$ \\
\hline Alternance & $\begin{array}{l}\text { Passage d'une situation/tâche globale à un élément spécifique et retour à la } \\
\text { tâche globale }\end{array}$ \\
\hline Application & Mise en action à travers une situation d'apprentissage \\
\hline Distinction & $\begin{array}{l}\text { Différenciation entre le contenu à apprendre et les processus sous-tendant } \\
\text { cet apprentissage }\end{array}$ \\
\hline Signifiance & $\begin{array}{l}\text { Utilisation de situations motivantes et intéressantes pour l'étudiant, reflétant } \\
\text { une situation authentique }\end{array}$ \\
\hline Cohérence & $\begin{array}{l}\text { Une relation cohérente entre les activités d'enseignements, les activités } \\
\text { d'apprentissages, les activités d'évaluation et les compétences à développer }\end{array}$ \\
\hline Intégration & $\begin{array}{l}\text { Appropriation des différentes composantes d'une compétence d'une façon } \\
\text { intégrée }\end{array}$ \\
\hline Itération & $\begin{array}{l}\text { Répétition d'un même contenu ou d'un même type de tâche favorisant } \\
\text { l'apprentissage }\end{array}$ \\
\hline Transfert & $\begin{array}{l}\text { Utilisation dans un autre contexte de connaissances et de capacités apprises } \\
\text { dans un contexte donné }\end{array}$ \\
\hline
\end{tabular}

Ces principes permettent à l'enseignant et au formateur clinique de réfléchir à la MEA la plus appropriée selon les connaissances antérieures à mobiliser, les nouveaux savoirs à acquérir, le niveau de l'apprenant, ainsi que le contexte associé. La plupart des MEA peuvent être adaptés au milieu clinique : histoires de cas, apprentissages par problèmes, activité de raisonnement clinique, pratique réflexive, laboratoire, jeu de rôle, simulation, etc. Par exemple, une activité de développement professionnel en milieu clinique pourrait être élaborée en débutant par la présentation d'une histoire de cas complexe (globalité et signifiance), puis en questionnant les apprenants sur leurs connaissances antérieures (construction). Tout au long de la formation, les apprenants seraient encouragés à poser des questions et faire des liens avec leur propre pratique clinique (signifiance et construction). La formation pourrait se terminer par un jeu de rôle ou une activité de simulation (application), permettant aux apprenants de mettre en pratique leurs nouveaux apprentissages. Tout au long de l'activité, le formateur s'assure du lien entre le contenu, les compétences à développer et le niveau des apprenants (cohérence).

Considérant qu'une compétence est toujours liée à un contexte de pratique (Tardif, 2006) et considérant le principe de signifiance, les activités de formation devraient être basées sur des mises en situation authentiques et d'une complexité croissante, adaptée au niveau des apprenants. Ceci peut amener un certain défi en milieu clinique, puisque les 
apprenants peuvent être de niveaux plutôt variés. Cependant, plus les enseignants et les formateurs cliniques gardent en tête ces principes lors du développement d'activités de formation, plus ils favorisent un apprentissage significatif.

Puisque l'action est au cœur de la définition d'une compétence, le développement des compétences est intimement lié à leur déploiement en contexte réel, qui peut être effectué pendant la formation (par exemple, lors d'un stage clinique ou en simulation haute-fidélité) ou à la suite de la diplomation, en milieu clinique. Même en étant novices, les étudiants peuvent expérimenter cette pratique infirmière; cette pratique est alors supervisée, selon le niveau de l'apprenant. Les concepts de développement et de déploiement de compétences ne doivent donc pas être interprétés comme linéaires ou survenant un à la suite de l'autre, mais plutôt interdépendants et s'influençant mutuellement. Par la réflexion dans l'action et sur l'action (Schön, 1993), l'étudiant ou le professionnel poursuit le développement de ses compétences, ce qui l'amène à des niveaux d'expertise plus élevés. Cette réflexion est essentielle et peut être faite de façon individuelle, par exemple en complétant un journal réflexif, ou collective, par exemple en faisant une séance de débriefing d'une activité de groupe.

Pour atteindre des niveaux de développement de compétences et d'expertise plus élevés, les infirmiers s'appuient à la fois sur des savoirs expérientiels et des savoirs théoriques qui, lorsque combinés, leur permettent de déployer leurs compétences plus efficacement. Ils seront alors en mesure d'adapter leurs interventions aux spécificités de chaque situation, de capturer les éléments significatifs et d'adapter leur pratique à l'unicité de chaque patient (Benner, 1984).

\section{Référentiel de compétences et niveau attendu de compétences}

Pour aider les formateurs cliniques à concevoir un programme ou une activité cohérente avec l'APC de seconde génération, un référentiel de compétences est non seulement utile, mais en devient la pierre angulaire (Poumay et Georges, 2017; Tardif, 2017). Un tel document est construit par une analyse du travail réel et attendu des professionnels et doit être validé empiriquement (Tardif, 2017). Il devrait inclure, pour chaque compétence, une définition précise, des connaissances à acquérir, des niveaux de développement ainsi que des indicateurs permettant d'apprécier la progression de l'apprenant (Poumay et George, 2017). Le nombre de compétences devrait être relativement restreint, considérant leur complexité et leur caractère intégrateur (Tardif, 2006). Ceci est cohérent avec les travaux du regroupement Quality and Safety Education for Nurses (Cronenwett et al., 2007), qui a développé un référentiel de six compétences basées sur les recommandations de l'IOM (2003) : 1) pratique centrée sur le patient, 2) collaboration interprofessionnelle et travail en équipe, 3) pratique basée sur les résultats probants, 4) amélioration de la qualité, 5) sécurité et 6) informatique et technologies.

L'enseignant ou le formateur clinique devrait utiliser le référentiel pour guider sa réflexion sur le type d'activités à élaborer, en fonction des intentions pédagogiques qu'il souhaite atteindre ou des compétences à développer. Les niveaux attendus de développement de compétences illustrent l'objectif à atteindre pour l'apprenant et l'objectif d'évaluation pour l'enseignant. Alors que l'élaboration de référentiels de compétences est de plus en plus fréquente dans des programmes de formation initiale en sciences infirmières, encore peu d'établissements de santé emboîtent le pas dans cette direction pour soutenir le développement professionnel des infirmières en exercice. Puisque le développement de compétences ne s'arrête pas à la diplomation (Benner, 1984; Tardif, 2006), les infirmiers pourraient bénéficier de ce type de document qui décrirait des niveaux attendus de compétences, en particulier pour les premières années de pratique. Par exemple, un 
programme de formation initiale d'une durée de trois ans pourrait avoir un référentiel de compétences qui détaillent trois niveaux attendus (fin de $1^{\text {ère }}$ année, fin de $2^{\mathrm{e}}$ année et fin de $3^{\text {e }}$ année) et ce, pour chacune des compétences. En milieu clinique, un référentiel de compétences pourrait être développé dans un même esprit, avec un niveau attendu après un an de pratique, trois ans et cinq ans.

Des auteurs ayant étudié les débuts professionnels d'infirmiers nouvellement diplômés concluent que le déploiement de leurs compétences s'améliore rapidement pendant la première année (Ke, Kuo, et Hung, 2017; Lima, Newall, Jordan, Hamilton, et Kinney, 2016). Ce déploiement ne se fait pas sans écueil; sans référentiel de compétences pour définir les compétences à développer et les niveaux attendus, des gestionnaires et formateurs cliniques expriment des attentes significativement différentes (Freeling et Parker, 2015; Numminen et al., 2014). Blanchet Garneau et al. (2017) précisent que cette confusion peut également provenir de l'interprétation que font certains individus de la visée d'une compétence : certains les interprètent comme un niveau minimal sécuritaire de soins et d'autres y voient plutôt une visée d'excellence. Cette dichotomie embrouille un discours déjà confus sur la définition d'une compétence, tel que précédemment discuté et provient, entre autres, de l'absence de référentiel de compétences dans de nombreux milieux de pratique.

\section{Une pratique infirmière ancrée dans un contexte}

À l'instar de Tardif et Benner, Charette et al. (2014) mettent l'accent sur l'importance du contexte lors du développement et du déploiement des compétences. Une compétence s'oriente toujours vers un but, une mise en action, une finalité; en sciences infirmières, cette finalité est le patient et la qualité des soins qui lui seront offerts. Cette finalité est tributaire du contexte de pratique dans lequel sont plongés les étudiants et les infirmiers (débutants ou expérimentés). Ce contexte comprend non seulement le milieu physique dans lequel ils se trouvent, mais également les professionnels y travaillant ainsi que les diverses politiques et règles mises en place. Ce contexte est dynamique; il évolue en fonction des professionnels qui le compose et des changements de politiques. En pratiquant dans un milieu clinique, les infirmiers influencent donc le contexte dans lequel ils se trouvent, lequel en retour aura une influence sur le comportement des infirmiers, ainsi que sur les apprentissages qu'ils pourront en tirer.

Des chercheurs (Numminen, Leino-Kilpi, Isoaho, et Meretoja, 2015) ont identifié des facteurs organisationnels ayant une influence statistiquement significative sur le déploiement de compétences des infirmiers (climat éthique et contexte de pratique), en plus de facteurs individuels (empowerment personnel et engagement professionnel). Charette et al. (2019b) ont également identifié des facteurs organisationnels et individuels, différents de ceux de Numminen et al. (2015). Ces facteurs organisationnels sont les caractéristiques de la période d'orientation, la stabilité sur une unité de soins et un quart de travail, la charge de travail et la culture scientifique des milieux de soins, alors que les facteurs individuels étaient la personnalité et l'expérience antérieure de stages des infirmiers nouvellement diplômés, le soutien ou l'intimidation manifestés par les autres infirmiers ainsi que la reconnaissance de l'expertise des infirmiers par les autres professionnels de la santé. Puisque ces facteurs influencent le contexte de pratique et le déploiement de compétences des infirmiers, les enseignants et formateurs cliniques ont tout intérêt à ne pas en faire abstraction, mais bien à s'en inspirer. De cette façon, on favorise l'application et le transfert de connaissances à ce contexte réel, imparfait et changeant. 


\section{Une qualité de soins optimale}

Le déploiement de compétences en contexte clinique vise à fournir des soins de qualité à la population. Le concept de qualité de soins est dynamique et composé de plusieurs dimensions, soit l'efficacité, l'efficience, l'accessibilité, l'acceptabilité (ou soins centrés sur le patient), l'équité ainsi que la sécurité (OMS, 2006).

Au-delà des concepts centraux reliés au développement et au déploiement de compétences, ce modèle met de l'avant la continuité et l'interrelation entre la formation et la pratique infirmière. Il pourrait donc être utilisé par les enseignants et les formateurs cliniques, en plus d'avoir des retombées pour la recherche qui seront discutées dans la prochaine section.

\section{Discussion}

Cet article présente un modèle appelant les enseignants et les formateurs cliniques vers une compréhension commune de l'interrelation entre le développement et le déploiement de compétences en pratique infirmière. Bien qu'il n'ait pas encore été validé empiriquement, une première étude qualitative a été effectuée en utilisant ce modèle comme cadre de référence (Charette et al., 2019a, 2019b). Cette étude a permis de corroborer certains liens en plus d'identifier des facteurs influençant le déploiement de compétences.

\section{Implications pour la recherche}

De nombreuses associations nationales qui règlementent la formation infirmière ont plaidé pour le développement de la recherche sur la formation infirmière. Des résultats empiriques sont nécessaires pour comprendre comment un programme axé sur les compétences prépare les infirmiers diplômés à pratiquer dans des systèmes de santé complexes et interprofessionnels. Les priorités de recherche de la National League of Nursing pour 2016-2019 plaident pour le développement de méthodes permettant de mesurer les résultats de programmes et les liens avec les soins aux patients. Dans un même ordre d'idées, l'Association canadienne des écoles de sciences infirmières (2014) encourage le développement de la recherche en éducation infirmière afin de promouvoir les connaissances disciplinaires et la contribution aux soins de santé de qualité. Il importe donc de fournir aux responsables de programmes de formation en sciences infirmières des connaissances issues de la recherche leur permettant de poursuivre le développement et l'amélioration des programmes de formation.

Or, encore peu de connaissances empiriques sont disponibles pour renseigner sur les retombées des programmes réformés sur l'APC, entre autres en termes de compétences déployées par les infirmiers diplômés de ces programmes (Morcke, Dornan, et Eika, 2013). La réforme de programmes en sciences infirmières représente une des réponses amenées par certaines maisons d'enseignement aux recommandations d'organismes nationaux et internationaux pour améliorer la qualité des soins. Toutefois, ces programmes innovateurs exigent d'importants efforts d'adaptation de la part des étudiants et des enseignants à une approche pédagogique très différente, sans compter la mobilisation d'importantes ressources humaines et financières (Goudreau et al., 2009). Le modèle de développement et déploiement de compétences en pratique infirmière invite les chercheurs à se questionner sur l'influence du contexte dans lequel exerce ces infirmiers et à tenir compte de ce contexte lorsqu'ils étudient le phénomène du développement et déploiement de compétences. Selon Pepin, Ducharme, et Kérouac (2017, p. 16), la discipline infirmière s'intéresse au "caractère entier de la personne [...] de même que l'interaction continue de chacune avec l'environnement interne $[\ldots]$ et externe $[\ldots]$ afin de bien comprendre l'expérience de santé ». Négliger de considérer l'influence du contexte sur l'action de la personne semble donc incompatible avec 
le centre d'intérêt holistique de la discipline, en plus d'être incohérent avec la définition de compétence de Charette et al. (2014).

Finalement, ce modèle invite les chercheurs à réfléchir au lien entre la formation des infirmiers et le déploiement de leurs compétences, rarement présent dans les études.

\section{Implications pour la formation initiale et continue}

En intégrant des concepts des sciences de l'éducation et des sciences infirmières, ce modèle de développement et de déploiement des compétences en pratique infirmière pourrait soutenir les enseignants et les formateurs dans le développement de MEA actives et basées sur des situations de soins réalistes et authentiques. Plus spécifiquement, les enseignants en formation initiale pourraient utiliser ce modèle pour mieux comprendre comment préparer les étudiants à agir en contexte réel. Puisqu'une compétence est une symbiose d'habiletés mises en action (Charette et al., 2014), il n'est pas suffisant d'avoir des connaissances déclaratives ou procédurales, mais les étudiants doivent également être en mesure d'agir en situation réelle et complexe. Le modèle permet de considérer les facteurs influençant le déploiement de compétences afin de préparer les étudiants à la réalité des milieux cliniques.

Ce modèle souligne également la nécessité d'un référentiel de compétences pour guider le développement des programmes de formation initiale ou de développement professionnel continu. Un tel document favorise la cohérence d'un cours à l'autre, tout au long du programme, et indique clairement les attentes. Bien que chaque établissement d'enseignement construit son propre référentiel, les compétences mises de l'avant sont souvent similaires et cohérentes avec le cadre développé par le regroupement Quality and Safety Education for Nurses (Cronenwett et al., 2007). Dans les milieux cliniques, une réflexion s'impose quant à l'adoption d'un référentiel de compétences, puisque plusieurs options sont possibles. Si un milieu jouit d'un partenariat avec une maison d'enseignement ayant un référentiel, l'option la plus simple est d'utiliser le même référentiel en ajoutant des niveaux attendus de développement de compétences après la diplomation. Une autre option pourrait être de développer un référentiel en s'inspirant d'un modèle existant, en l'adaptant aux spécificités du milieu clinique.

Peu importe l'option retenue, il est essentiel d'établir pour chaque compétence quelques niveaux attendus de développement. Autant pour la formation initiale que la formation continue, l'identification de ces niveaux devrait se faire empiriquement, par la construction d'un modèle cognitif d'apprentissage (MCA) pour chaque compétence (Tardif, 2006). Ces MCA identifient les apprentissages critiques que les apprenants doivent effectuer afin de franchir les étapes de développement d'une compétence, représentant un processus de réorganisation cognitive (Tardif, 2017).

Pour les formateurs des milieux cliniques, ce modèle de développement et déploiement de compétences en pratique infirmière met en évidence l'interdépendance entre la théorie et la pratique, ainsi que l'importance de miser sur des MEA actives pour favoriser le développement des compétences. Les formateurs cliniques pourraient également utiliser un tel modèle pour considérer les facteurs organisationnels et individuels dans l'élaboration de programmes d'accueil et d'orientation d'infirmiers nouvellement diplômés ou pour développer des outils facilitant le suivi du développement et déploiement des compétences.

\section{Limites}

La principale limite de ce modèle de développement et de déploiement de compétence en pratique infirmière est qu'il est le résultat d'une intégration théorique. De futures études seront nécessaires afin de le valider empiriquement. 


\section{Conclusion}

Le modèle de développement et de déploiement de compétences en pratique infirmière présenté dans cet article constitue un travail d'intégration de deux approches souvent citées en formation en sciences infirmières, soit l'APC de seconde génération, provenant des sciences de l'éducation, et les travaux de Patricia Benner sur le développement de l'expertise professionnelle. Ce modèle vise le partage d'un langage commun pour les enseignants et les formateurs cliniques en sciences infirmières. Ce modèle permet de considérer que le développement des compétences ne s'arrête pas à la diplomation et que toutes les activités de formation devraient être ancrées dans un contexte authentique, soit des situations de soins reflétant la réalité d'une pratique professionnelle. D'autres études seront nécessaires afin de bonifier ou de valider empiriquement ce modèle de développement et de déploiement de compétences en pratique infirmière. Cependant, son l'utilisation pourrait faciliter un dialogue entre les maisons d'enseignement et les établissements de santé, les formateurs cliniques et les gestionnaires. Ce dialogue devient nécessaire afin de soutenir l'intégration des infirmiers en début de pratique, favorisant le déploiement optimal de leurs compétences afin d'assurer des soins de qualité pour la population. 


\section{Références}

Association canadienne des écoles de sciences infirmières (ACÉSI). (2014). Plan stratégique 2014-2018. Repéré à http://www.casn.ca/vm/newvisual/attachments/856/Media/ StratplanBillingual.pdf

Benner, P. (1984). From novice to expert: excellence and power in clinical nursing practice. Menlo Park, CA: Addison-Wesley Publishing.

Benner, P., Sutphen, M., Leonard, V., et Day, L. (2010). Educating Nurses: A Call for Radical Transformation. San Francisco, CA: Jossey-Bass.

Benner, P., Tanner, C., et Chesla, C. (1992). From beginner to expert: gaining a differentiated clinical world in critical care nursing. Advances in Nursing Science, 14(3), 13-28. https://doi.org/10.1097/00012272-199203000-00005

Billings, D. M., et Halstead, J. A. (2016). Teaching in Nursing: A Guide for Faculty (5éd.). St-Louis, MO: Elsevier.

Benner, P., Tanner, C., et Chesla, C. (2009). Expertise in Nursing Practice: Caring, Clinical Judgment, and Ethics. ( $2^{\mathrm{e}}$ éd.). New York, NY: Springer Publishing Company.

Blanchet Garneau, A., Lavoie, P., et Grondin, M. (2017). Dichotomy and dialogue in conceptualizations of competency in health professionals' education. Journal of Nursing Education and Practice, 7(6), 18-26. https://doi.org/10.5430/jnep.v7n6p18

Boulet, A., Savoie-Zajc, L., et Chevrier, J. (1996). Les stratégies d'apprentissages à l'université. Québec, QC: Presses de l'Université du Québec.

Charette, M., Goudreau, J., et Alderson, M. (2014). Une analyse évolutionniste du concept de compétence. Recherche En Soins Infirmiers, (116), 27-39. https://doi.org/10.3917/rsi.116.0028

Charette, M., Goudreau, J., \& Bourbonnais, A. (2019a). How do new graduate nurses from a competency-based program demonstrate their competencies? A focused ethnography of acute care settings. Nurse Education Today, 79, 161-167. https://doi.org/10.1016/j.nedt.2019.05.031

Charette, M., Goudreau, J., \& Bourbonnais, A. (2019b). Organizational and individual factors influencing the practice of new graduate nurses: a focused ethnography of acute care settings. Journal of Clinical Nursing, 28(19/20), 3618-3631.

https://doi.org/10.1111/jocn.14959

Cronenwett, L., Sherwood, G., Barnsteiner, J., Disch, J., Johnson, J., Mitchell, P., . . Warren, J. (2007). Quality and safety education for nurses. Nursing Outlook, 55(3), 122-131. https://doi.org/10.1016/j.outlook.2007.02.006

Dadgaran, I., Parvizy, S., et Peyrovi, H. (2012). A global issue in nursing students' clinical learning: the theory-practice gap. Procedia-Social and Behavioral Sciences, 47, 17131718. https://doi.org/10.1016/j.sbspro.2012.06.888

Dewey, J. (1997). Experience and education. New York, NY: Simon \& Schuster.

Dreyfus, S. E., et Dreyfus, H. L. (1980). A five-stage model of the mental activities involved in directed skill acquisition. Berkeley, CA: University of California.

Duchscher, J. B. (2009). Transition shock: the initial stage of role adaptation for newly graduated Registered Nurses. Journal of Advanced Nursing, 65(5), 1103-1113. https://doi.org/10.1111/j.1365-2648.2008.04898.x 
Flexner, A. (1910). Medical education in the United Stated and Canada. Repéré à http://www.carnegiefoundation.org/sites/default/files/elibrary/ Carnegie_Flexner_Report.pdf

Franklin, N., \& Melville, P. (2015). Competency assessment tools: An exploration of the pedagogical issues facing competency assessment for nurses in the clinical environment. Collegian, 22(1), 25-31. https://doi.org/10.1016/j.colegn.2013.10.005

Freeling, M., et Parker, S. (2015). Exploring experienced nurses' attitudes, views and expectations of new graduate nurses: A critical review. Nurse Education Today, 35(2), e42-e49. https://doi.org/10.1016/j.nedt.2014.11.011

Frenk, J., Chen, L., Bhutta, Z. A., Cohen, J., Crisp, N., Evans, T., ... Zurayk, H. (2010). Health professionals for a new century: transforming education to strengthen health systems in an interdependent world. The Lancet, 376(9756), 1923-1958. https://doi.org/10.1016/s0140-6736(10)61854-5

Garside, J. R., et Nhemachena, J. Z. Z. (2013). A concept analysis of competence and its transition in nursing. Nurse Education Today, 33(5), 541-545. https://doi.org/10.1016/j.nedt.2011.12.007

Goldmark, J. (1923). Nursing and nursing education in the United States. Repéré à https://archive.org/details/nursingnursinged00comm

Gonczi, A. (1996). Reconceptualising competency-based education and training: with particular reference to education for occupations in Australia (Thèse de doctorat). University of Technology of Sydney.

Gonczi, A., et Hager, P. (2010). The competency model. Dans P. Peterson, E. Baker et B. McGaw (dir.), International Encyclopedia of Education (Vol. 8). Oxford, RoyaumeUni: Elsevier.

Goudreau, J., Pepin, J., Dubois, S., Boyer, L., Larue, C., et Legault, A. (2009). A second generation of the competency-based approach to nursing education. International Journal of Nursing Education Scholarship, 6(1), Article 15. https://doi.org/10.2202/1548-923x.1685

Guillemette, F., et Gauthier, C. (2008). L'approche par compétences (APC) en formation des maîtres: Analyse documentaire et critique. Recherches \& éducations. Repéré à http://journals.openedition.org/rechercheseducations/84

Hatlevik, I. K. R. (2012). The theory-practice relationship: reflective skills and theoretical knowledge as key factors in bridging the gap between theory and practice in initial nursing education. Journal of Advanced Nursing, 68(4), 868-877. https://doi.org/10.1111/j.1365-2648.2011.05789.x

Institute of Medicine (2003). Health Professions Education: A Bridge to Quality. Washington, D.C.: The National Academies Press.

Ishler, R. E. (1974). Competency-based teacher education: A potpourri of perspectives. Washington, D.C.: Association of Teacher Educators.

Ke, Y.-T., Kuo, C.-C., et Hung, C.-H. (2017). The effects of nursing preceptorship on new nurses' competence, professional socialization, job satisfaction and retention: A systematic review. Journal of Advanced Nursing, 73, 1-10. https://doi.org/10.1111/jan.13317

Lasnier, F. (2000). Réussir la formation par compétences. Montréal, QC: Guérin. 
Lima, S., Newall, F., Jordan, H. L., Hamilton, B., et Kinney, S. (2016). Development of competence in the first year of graduate nursing practice: a longitudinal study. Journal of Advanced Nursing, 72(4), 878-888. https://doi.org/10.1111/jan.12874

Meretoja, R., et Koponen, L. (2012). A systematic model to compare nurses' optimal and actual competencies in the clinical setting. Journal of Advanced Nursing, 68(2), 414422. https://doi.org/10.1111/j.1365-2648.2011.05754.X

Morcke, A. M., Dornan, T., et Eika, B. (2013). Outcome (competency) based education: an exploration of its origins, theoretical basis, and empirical evidence. Advances in Health Sciences Education, 18, 851-863. https://doi.org/10.1007/s10459-012-9405-9

Morgan, D. L. (2014). Pragmatism as a Paradigm for Social Research. Qualitative Inquiry, 20(8), 1045-1053. https://doi.org/10.1177/1077800413513733

National League for Nursing. (2016). NLN Research Priorities in Nursing Education 20162019. Repéré à http://www.nln.org/docs/default-source/professional-developmentprograms/nln-research-priorities-in-nursing-education-single-pages.pdf?sfvrsn=2

Numminen, O., Laine, T., Isoaho, H., Hupli, M., Leino-Kilpi, H., et Meretoja, R. (2014). Do educational outcomes correspond with the requirements of nursing practice: educators' and managers' assessments of novice nurses' professional competence. Scandinavian Journal of Caring Sciences, 28, 812-821. https://doi.org/10.1111/scs.12115

Numminen, O., Leino-Kilpi, H., Isoaho, H., et Meretoja, R. (2015). Newly Graduated Nurses' Competence and Individual and Organizational Factors: A Multivariate Analysis. Journal of Nursing Scholarship, 1-12. https://doi.org/10.1111/jnu.12153

Organisation mondiale de la Santé. (2006). Quality of Care: A Process for making Strategic Choices in Health Systems. Retrieved from http://www.who.int/management/quality/assurance/ QualityCare_B.Def.pdf

Pastré, P. (1999). La conceptualisation dans l'action: bilan et nouvelles perspectives. Éducation permanente, 139, 13-35.

Pepin, J., Ducharme, F., et Kérouac, S. (2017). La pensée infirmière (4éd.). Montréal, QC: Chenelière Éducation.

Perrenoud, P. (2004). Adosser la pratique réflexive aux sciences sociales, condition de la professionnalisation. Dans J.-F. Inisan (dir.), Analyse de pratiques et attitude réflexive en formation (p. 11-32). Reims, France: CRDP de Champagne-Ardenne.

Polit, D. F., et Beck, C. T. (2017). Nursing research : generating and assessing evidence for nursing practice (10 éd.). Philadelphia, PA: Wolters Kluwer Health.

Poumay, M., et Georges, F. (2017). Des balises méthodologiques pour construire un référentiel de compétences et une grille de programme. Dans M. Poumay, J. Tardif et F. Georges (dir.), Organiser la formation à partir des compétences : Un pari gagnant pour l'apprentissage dans le supérieur. Louvain-la-Neuve, Belgique: De Boeck Supérieur.

Rischel, V., Larsen, K., et Jackson, K. (2008). Embodied dispositions or experience? Identifying new patterns of professional competence. Journal of Advanced Nursing, 61(5), 512-521. https://doi.org/10.1111/j.1365-2648.2007.04543.x

Risjord, M. (2010). Nursing Knowledge. Science, Practice and Philosophy. Chichester, Royaume-Uni: Wiley-Blackwell. 
Saifan, A., AbuRuz, M. E., et Masa'deh, R. (2015). Theory Practice Gaps in Nursing Education: A Qualitative Perspective. Journal of Social Sciences/Sosyal Bilimler Dergisi, 11(1), 22-29. https://doi.org/10.3844/jssp.2015.20.29

Schön, D. A. (1993). Le praticien réflexif. À la recherche du savoir caché dans l'agir professionnel. Montréal, QC: Les Éditions Logiques.

Shabani, K., Khatib, M., \& Ebadi, S. (2010). Vygotsky's Zone of Proximal Development: Instructional Implications and Teachers' Professional Development. English Language Teaching, 3(4), 237-248. https://doi.org/10.5539/elt.v3n4p237

Smith, S. A. (2012). Nurse Competence: A Concept Analysis. International Journal of Nursing Knowledge, 23(3), 172-182. https://doi.org/10.1111/j.2047-3095.2012.01225.x

Steiner, G. (2001). Transfer of Learning, Cognitive Psychology of. Dans N. J. Smelser et P. B. Baltes (Éds.), International Encyclopedia of the Social \& Behavioral Sciences $\left(2^{\mathrm{e}}\right.$ éd., p. 15845-15851). Oxford, Royaume-Uni: Pergamon.

Tardif, J. (2006). L'évaluation des compétences - Documenter le parcours de formation. Montréal, QC: Chenelière Éducation.

Tardif, J. (2016). Ancrer les apprentissages sur les connaissances antérieures des étudiants. Dans T. Pellascia (dir.), Comment (mieux) évaluer et former les étudiants en médecine et en sciences de la santé?. Louvain-la-neuve, Belgique: De Boeck Supérieur.

Tardif, J. (2017). Des repères conceptuels à propos de la notion de compétence, de son développement et de son évaluation. Dans M. Poumay, J. Tardif et F. Georges (dir.), Organiser la formation à partir des compétences : Un pari gagnant pour l'apprentissage dans le supérieur. Louvain-la-Neuve, Belgique: De Boeck Supérieur.

Weir, G. M. (1932). Survey of Nursing Education in Canada. Vancouver, BC: University of British Columbia. 


\section{Introduction}

Educational institutions offering pre-licensure (or entry-level) nursing programs are constantly reforming them to provide quality education and to meet the needs of the population. Unfortunately, communication is often deficient between these institutions and clinical settings (Saifan, AbuRuz, \& Masa'deh, 2015), creating a confusion and a feeling of unpreparedness for new graduate nurses (NGNs) who are in a transition from the student to health professional role (Duchscher, 2009). Moreover, academics in educational institutions and clinical educators in health care organizations do not always share the same vision of competency (Blanchet Garneau, Lavoie, \& Grondin, 2017), which adds confusion to an already challenging situation. Dadgaran, Parvizy, and Peyrovi (2012) suggest that the transformation of pre-licensure programs could enhance the communication between the academic and clinical worlds, if such programs would use an approach based on advanced practical teaching-learning methods (e.g., high-fidelity simulation, clinical placement and hands-on lab sessions), while also promoting the interdependence of practice and theoretical knowledge.

Competency-based education (CBE) meets these criteria. The Institute of Medicine (IOM; 2003) concluded that the transformation of health professional education programs into $\mathrm{CBE}$ would better prepare students for the challenges of future health care systems as well as promoting interprofessional collaboration. This major change could address the suboptimal quality of care provided to patients, caused in part by inadequate pre-licensure education of health professionals, as concluded by the IOM.

$\mathrm{CBE}$, as promoted by the IOM, requires that learning be viewed as an active and contextualized process, preparing students to act in real-life situations. The use of active teaching and learning methods (TLM), such as problem-based learning or high-fidelity simulation, is the result of a paradigm shift from teaching to learning, thus from passive didactic pedagogy centered on the transmission and acquisition of knowledge towards an active approach where each student holds the key to his/her own learning (Tardif, 2006). In this approach, competencies are holistic: authors in both educational sciences (Gonczi, 1996; Gonczi \& Hager, 2010; Lasnier, 2000; Poumay \& Georges, 2017; Tardif, 2006) and nursing (Blanchet Garneau et al., 2017; Charette, Goudreau, \& Alderson, 2014; Garside \& Nhemachena, 2013; Smith, 2012) have defined a competency as complex know-how or a strategic combination of knowledge, skills and attitude, bound to a context and continually evolving.

$\mathrm{CBE}$ invites us to place students at the heart of their learning processes through action (Tardif, 2006). Other authors have stated that reflection-in-action and reflection-on-action as being as important as the action itself (Pastré, 1999; Perrenoud, 2004; Schön, 1984) and that the development of reflective skills increases the perception of coherence between theoretical knowledge and practical skills (Hatlevik, 2012). Reflective activities can be implemented in a pre-licensure program for students or in a continuing education program for registered nurses.

While CBE has been adopted by many educational institutions for pre-licensure nursing programs, some health care organizations have also followed the trend by developing CBE-based continuing education and transition-to-practice programs for NGNs. In addition, the use of a common language shared by academics and clinical educators, particularly in terms of the development and deployment of competencies, would make it possible to situate expectations for students and NGNs on a continuum, beginning during pre-licensure and continuing in the clinical setting. Collaboration between the academic and clinical communities can thus become a cornerstone for knowledge transfer and the integration of learning, fostering quality nursing practice. A theoretical model linking educational theories 
of learning to nursing practice in health care settings could potentially provide this common language. This article describes such a model for competency development and deployment in nursing practice.

\section{Theoretical Underpinnings}

This theoretical model was established through the integration of CBE with Benner's work on the development of professional expertise in nursing practice. These two foundations will be described in this section.

\section{Competency-based Education}

In the last century, three major reforms have transformed health care education programs. A major reformation in the early 1900s led to the creation of medical and nursing programs based on the acquisition of biomedical sciences knowledge (Flexner, 1910). Pre-dating such programs, in the mid- $19^{\text {th }}$ century, Florence Nightingale, a strong advocate for the scientific training of nurses, had created the first independent hospital nursing school. In a 1932 report, Weir argued that nursing should be regarded as a profession, rather than under the hegemony of medicine, and thus, that knowledge taught to nurses should be adapted to their own professional context.

Towards the 1950s, educators began to promote the idea that simple transmission of knowledge by experts was no longer sufficient for preparing competent professionals (Guillemette \& Gauthier, 2008). This reflection led to a second major reform, in which the proper use of knowledge became as important, if not more important, than knowledge acquisition. This reform was marked by the introduction of active TLMs (such as problembased learning) in some pre-licensure programs for health professionals (Frenk et al., 2010). The term competency also took on a connotation of performance (Tardif, 2006). Competency frameworks were developed to support these programs, generally through behavioral analysis of the professional's work, focused on performing tasks in both simple and complex situations. This meticulous analysis gave rise, in some cases, to frameworks composed of hundreds or even thousands of competencies (Ishler, 1974).

This first-generation CBE was criticized, the main criticism being that it prioritized technical performance over cognitive internal processes linked to the professional's actions and learning. The emphasis on specific behaviors has led some authors to label it behaviorist CBE (Guillemette \& Gauthier, 2008). Reflections led to a second-generation CBE based on cognitivism and constructivism (Gonczi \& Hager, 2010; Goudreau et al., 2009). While firstgeneration $\mathrm{CBE}$ highlighted the importance of the actions and performance of professionals, the second generation considered a competency to integrate both an action and the cognitive processes that underlie it, through the complexity of the situations experienced by the professional (Tardif, 2006).

Cognitivism, a theory from modern psychology, is primarily the study of how a person acquires and uses knowledge, by having information processed through short-term memory (also known as working memory) and long-term memory. The different types of knowledge that students acquire (theoretical, technical and conditional knowledge; Boulet, Savoie-Zajc, \& Chevrier, 1996) can subsequently be mobilized and combined, allowing students to act competently in a variety of situations (Tardif, 2006). The other theory on which CBE is based is constructivism (Billings \& Halstead, 2016) which is the integration of new knowledge into previous knowledge, through the construction of complex cognitive schemas (Tardif, 2016); this gives the learner an increasingly large repertoire of actions and the ability to transfer his/her learning in various situations (Steiner, 2001). Vygotsky added a social dimension to the construction of knowledge, in what is referred to as 
socioconstructivism (Tardif, 2016). Through exchanges with his peers, a learner's cognitive schemas are shaken up and enriched by a process of deconstruction and reconstruction. Vygotsky also introduced the notion of the zone of proximal development, which represents what a learner cannot do alone but can achieve with the help of someone else (Shabani, Khatib \& Ebadi, 2010). A teacher and fellow students therefore have a primary role in an individual's learning process; the teacher must vary activities and learning opportunities to encourage exchanges between individuals.

Considering these two theories (three if we consider socioconstructivism to be a separate theory), second-generation CBE promotes learning by pushing students to be active players in their own education. By using a variety of TLMs, the teacher tries to foster a deeper understanding and integration of different types of knowledge and the transfer of learning into many complex and varied situations, rather than aiming for memorization of a great amount of knowledge (Tardif, 2006). In a health care context, the goal of these CBE programs is to prepare self-reflective competent practitioners, ready to tackle the realities of present and future health care systems (Frenk et al., 2010). The development of core competencies, adapted to the professional context of each discipline, could also promote the interprofessional collaboration needed to meet the needs of the population.

In the educational sciences literature, several definitions of competency can be found and classified according to a behaviorist vision, coherent with first-generation CBE, or to a holistic vision, coherent with second-generation CBE. The behavioral definition of competency refers to the notion of performance and specific, measurable outcomes (Tardif, 2006). The holistic vision of competency encompasses a range of knowledge, skills and attitudes that a person possesses, and which allow him/her to be competent, in addition to identifying that one's competencies are intimately related to the context in which they evolve (Gonczi \& Hager, 2010).

A critical analysis of the concept of competence in nursing education was conducted (Charette et al., 2014), revealing that most authors use a holistic definition of competency. This analysis of 41 papers published between 2008 and 2013 identified essential characteristics and consequences of the concept of competence. According to these authors, a competency is a process of systemic skills, context-bound and continually evolving. In this definition, systemic skills refer to a symbiosis of psychomotor, cognitive and affective skills, similar to the notion of complex know-how (Tardif, 2006) or knowledge, skills and attitude (Cronenwett et al., 2007). The authors also identified that when a nurse practices competently, positive consequences such as public protection, job satisfaction and dialogue between practitioners occur (Charette et al., 2014).

Finally, several authors discussed competency assessment in nursing education (Garside \& Nhemachena, 2013; Meretoja \& Koponen, 2012; Rischel, Larsen, \& Jackson, 2008). These authors stated that in order to be consistent with a holistic definition competency, nurses should not be considered competent solely based on their technical skills (e.g., assessment of rapidity), which is only one aspect of nursing practice. Because the concept of competency is complex and multi-faceted, evaluation should reflect this complexity (Franklin \& Melville, 2015). These authors, similar to Tardif (2006), recommend that the evaluation be carried out at several moments in order to account for a learner's development trajectory rather than a snapshot.

In summary, the second-generation $\mathrm{CBE}$, based on cognitivism and constructivism, allows learning to be viewed as a process in which the student is active. Various TLMs will be developed by the teacher to help the student acquire different types of knowledge that will 
enable him/her to act in authentic professional situations and develop his/her professional expertise.

\section{Professional Expertise: From novice to expert}

Patricia Benner has studied the phenomenon of professional expertise development by adapting Dreyfus and Dreyfus' skills acquisition model (Dreyfus \& Dreyfus, 1980) to nursing. In a multi-center phenomenological study ( $\mathrm{n}=62$ nurses and 5 nursing students), Benner (1984) described five stages of expertise development (see Table 1) and explained that the change from one stage to another is not an automatic process but occurs only if the nurse engages in a continuous process of experiential learning. Although the expert stage is the last stage, it should not be regarded as the end of the process, but rather as a period where the nurse continues to develop her/his expertise by incorporating new knowledge into that experience.

Table 1

Brief description of each stage from novice to expert (inspired from Benner, 1984)

\begin{tabular}{|c|l|}
\hline Stage & \multicolumn{1}{c|}{ Brief description } \\
\hline Novice & $\begin{array}{l}\text { The novice has no clinical experience and lacks confidence. He/she uses rules } \\
\text { to guide his/her actions, which are limited and applied universally. }\end{array}$ \\
\hline $\begin{array}{c}\text { Advanced } \\
\text { beginner }\end{array}$ & $\begin{array}{l}\text { The advanced beginner shows acceptable performance, but often feels } \\
\text { overwhelmed and incompetent. Heshe has a hard time organizing or } \\
\text { prioritizing his/her actions but demonstrate an acceptable performance. }\end{array}$ \\
\hline Competent & $\begin{array}{l}\text { The competent nurse can draw back on his/her experience to organize, plan } \\
\text { and deliver care. He/she identifies the most salient elements of a situation and } \\
\text { can react to complex situations. }\end{array}$ \\
\hline Proficient & $\begin{array}{l}\text { The proficient nurse perceives the situation in a holistic way. He/she can } \\
\text { easily distinguish salient elements and see subtle changes in situations. } \\
\text { His/sne decision-making process is more efficient. }\end{array}$ \\
\hline Expert & $\begin{array}{l}\text { The expert nurse has an intuitive grasp of every situation and a long-term } \\
\text { vision. He/she can easily adapt his/her practice to the uniqueness of each } \\
\text { situation. His/her performance is fluid and highly efficient. }\end{array}$ \\
\hline
\end{tabular}

While Benner's work is well known in nursing, there is some confusion about certain aspects. Over the years, Benner's work on the development of professional expertise has received many labels: novice-to-expert model and theory, stages of clinical competence, and nursing skill acquisition theory, to name just a few. However, from an epistemological point of view, models and theories are not equivalent, and different levels of theory exist (e.g., practical, middle-range or grand theory; Risjord, 2010). One reason for this confusion was a paradigmatic debate about the validity of interpretive research for the advancement of knowledge, a debate that reached its peak in the 1970 s and ' 80 s but seems to continue to this day. Benner was one of the first nursing researchers to study and describe nursing knowledge incorporated into practice, using a qualitative method. In doing so, she has established the usefulness of this paradigm for nursing research (Risjord, 2010). While her work was influential, it does not meet the level of abstraction required to be considered a theory and should rather be considered a theoretical model, since she has made it possible to describe a phenomenon and its associated stages, using empirical data (Risjord, 2010).

In addition to this plurality of titles, there is also confusion about the first two stages of Benner's model. Some have interpreted the novice learner as the NGN and have therefore 
concluded that the model is not concerned with nursing education, while others consider the novice learner to be the nursing student. While reporting the results of a qualitative study to deepen the model, Benner, Tanner, and Chesla (1992) specified that Benner's second stage (advanced beginner) corresponds to NGN. This may seem to be a trivial point, but it confirms that Benner's development of professional expertise begins during pre-licensure education and encompasses the transition into clinical settings.

Explicitly, the novice-to-expert model refers to skills acquisition, not competency development. However, Benner (1984) believed that these skills should be considered a holistic performance, bound to a practice setting, and that the actions of a nurse should not be considered strictly from a procedural point of view. Therefore, as previously stated, the way she described these skills is more consistent with the holistic definition of competency (Charette et al., 2014).

Recent publications by Benner highlight her pedagogical vision for nursing education (Benner, Sutphen, Leonard, \& Day, 2010; Benner, Tanner, \& Chesla, 2009). She advocates for a transformation of education programs towards an integrative pedagogy that allows for an interdependence between knowledge and experience or between theory and practice. Benner's reflections on nursing education (as well as her model) are based on Dewey's experiential learning (Dewey, 1997). A pragmatic American philosopher, Dewey promoted a progressive education: a pedagogy in which experience is the foundation of the student's knowledge and the motor of learning, and where this knowledge is used to generate human action (Morgan, 2014). In this philosophical stream, the focus is on the interaction between the learner and his/her environment (social, physical, cultural and historical). Learners develop predispositions and capacities to act that are bound to a context, also called situated or contextualized cognition.

Benner's pedagogical vision is based on this concept of situated cognition (Benner et al., 2009). As a nurse progresses in the development of her expertise, she becomes able to capture the significant elements of a situation (what Benner calls a sense of salience), to grasp the uniqueness of each situation and to adapt her practice to that situation. This process can only be achieved through a deep, meaningful and authentic patient-nurse relationship. To attain higher levels of expertise, Benner emphasizes the interdependence of research, theory, and practice (Benner et al., 2009): a nurse should not only rely on her experiential knowledge, but also on empirical knowledge, to reach her/his full potential.

\section{Model of competency development and deployment in nursing practice}

Many connections can be made between the development of professional expertise, Benner's educational vision, and second-generation CBE. Through the integration of these two approaches, a model of competency development and deployment in nursing practice (see Figure 1) was built to guide a qualitative study aimed at describing the competency deployment of NGNs in acute care settings (Charette, Goudreau, \& Bourbonnais, 2019a, $2019 \mathrm{~b}$ ). Benner's key concepts of contextualization, experiential learning, reflection on practice, and progression through different stages are also at the heart of $\mathrm{CBE}$, which makes it possible to operationalize these concepts in pre-licensure programs as well as in continuing education. 
Figure 1

Model of competency development and deployment in nursing practice

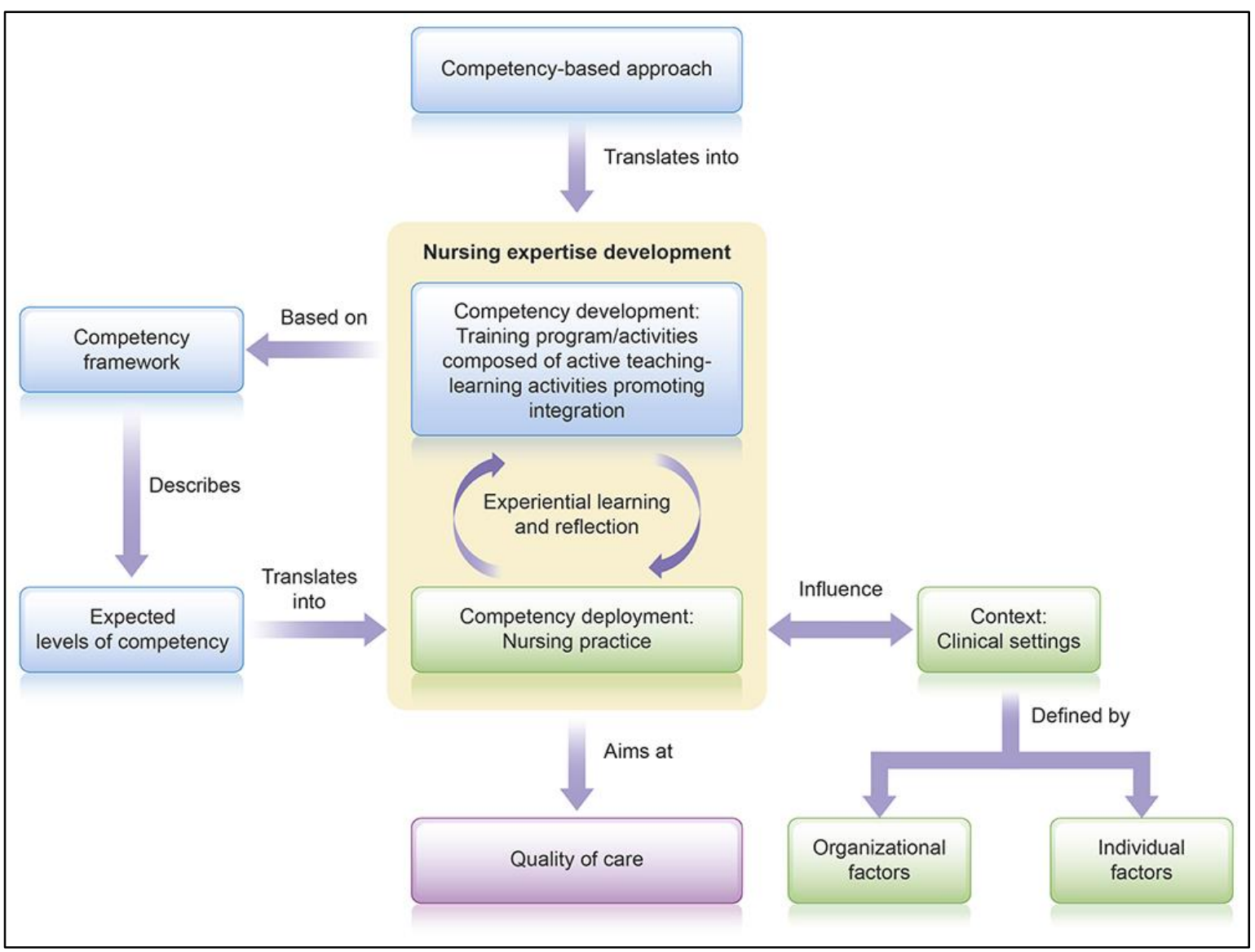

This model could guide academics and clinical educators in the development of activities in academic and health care settings, by pointing out essential elements: active TLMs based on authentic situations, the importance of reflection, the usefulness of a framework outlining competencies to develop and the level to which they should be developed, and factors influencing nursing practice. By illustrating the continuum between nursing education and practice, this model promotes the use of a common language and mindset. In doing so, NGNs should feel a stronger sense of coherence between the education they receive and the expectations of their employers, allowing for a smoother and easier transition into the professional field.

\section{Nursing Expertise Development}

At the heart of this model lies the concept of nursing expertise described by Benner (Benner, 1984; Benner et al., 2009), which encompasses both competency development and deployment. The term development refers to the ongoing process of acquiring and integrating various types of knowledge into one's cognitive schemas, whereas deployment is putting this symbiosis of skills into action in authentic situations to produce a result. In order to promote the development of students' (novice) competence and to be consistent with secondgeneration $\mathrm{CBE}$, a pre-licensure program or continuing education should consist of active TLMs (Benner et al., 2010; Tardif, 2006), such as problem-based learning, high-fidelity simulation or clinical placement. These activities should be planned according to pedagogical principles such as those described in Table 2. 
Table 2

Pedagogical principles (translated and inspired from Lasnier, 2000, pp. 158-174)

\begin{tabular}{|l|l|}
\hline \multicolumn{1}{|c|}{ Principle } & \multicolumn{1}{|c|}{ Brief description } \\
\hline Globality & $\begin{array}{l}\text { Tasks should be based on a global and complex situation to give the learner } \\
\text { an overview of what needs to be done }\end{array}$ \\
\hline Construction & $\begin{array}{l}\text { To facilitate the connection of new knowledge with previous knowledge, } \\
\text { previous knowledge needs to be re-activated first }\end{array}$ \\
\hline Alternation & $\begin{array}{l}\text { Moving from a global situation / task to a specific element and back to the } \\
\text { global task }\end{array}$ \\
\hline Application & $\begin{array}{l}\text { The learner should be given the possibility to act or apply the new } \\
\text { knowledge in an authentic situation }\end{array}$ \\
\hline Distinction & $\begin{array}{l}\text { Differentiation between the content to be learned and the processes } \\
\text { underlying this learning }\end{array}$ \\
\hline Significance & Use of interesting and authentic situations \\
\hline Coherence & $\begin{array}{l}\text { An explicit connection between teaching-learning activities, evaluation } \\
\text { methods and competencies to be developed }\end{array}$ \\
\hline Integration & The combination of knowledge, skills and attitude related to the competency \\
\hline Iteration & $\begin{array}{l}\text { Repetition of the same knowledge or of the same type of situation to } \\
\text { promote learning }\end{array}$ \\
\hline Transfer & Use of the same knowledge, skills and attitude in another context or situation \\
\hline
\end{tabular}

Academics and clinical educators should keep these principles in mind when choosing the most appropriate TLM and designing an activity. In other words, one should consider the prior knowledge to be mobilized, the new knowledge to be acquired, the level of the learner, and the context (both learning and clinical). Most TLMs can be adapted to the clinical setting: case study, problem-based learning, clinical reasoning activity, reflective practice, laboratory, role play, simulation, etc. For example, a continuing education session in a clinical setting could be developed starting with the presentation of a complex case study (globality and significance), and then questioning the learners about their previous knowledge (construction). Throughout the session, learners would be encouraged to ask questions and make connections with their own clinical practice (significance and construction). The session could end with a role play or a simulation activity (application), allowing learners to practice what they learned. Throughout the activity, the educator ensures the link between the content, the competency to develop and the level of learners (coherence).

Considering the contextualized nature of a competency and the principle of significance, learning activities should be based on authentic and increasingly complex scenarios, adapted to the level of the learners. This process can be a challenge in a clinical setting, as learners can be of varying levels. However, by keeping these principles in mind when developing activities, academics and clinical educators will promote meaningful learning.

The development of competencies is intimately linked to their deployment in an authentic context, whether during a pre-licensure program or post-graduation in a health care organization. As novices, students can either be placed in a simulated environment or a reallife situation (for example, in a clinical placement), through supervised practice adapted to their stage and knowledge. Through reflection in and on action (Schön, 1984), the learners (student or nurse) will integrate experiential and theoretical knowledge that, once combined, allows them to deploy their competencies more effectively, which in turn leads to higher levels of expertise. This reflection is essential and can be achieved individually, e.g., by 
completing a reflective journal, or collectively, e.g., by engaging in a group debriefing session.

The concepts of development and deployment of competencies should not be interpreted as linear or occurring one after the other, but rather as interdependently and mutually influencing one another. To achieve higher levels of competency and expertise development, nurses rely on both experiential knowledge and theoretical knowledge that, when combined, allows them to deploy their competencies more effectively. They will then be able to adapt their interventions to the specificities of each situation, to capture the significant elements and to adapt their practice to the uniqueness of each patient (Benner, 1984).

\section{Competency Framework and Expected Levels of Competency}

Having a well-defined competency framework can support academics and clinical educators in designing a program (or course or activity) consistent with second-generation CBE (Poumay \& Georges, 2017; Tardif, 2017); it is built from task analysis and should be empirically validated (Tardif, 2017). The number of competencies should be relatively low, in consideration of their complexity and integrability (Tardif, 2006). This is consistent with research by Quality and Safety Education for Nurses (Cronenwett et al., 2007), a collaborative group who designed a framework of six competencies based on IOM recommendations (Institute of Medicine, 2003) namely: 1) patient-centered practice; 2) teamwork and collaboration; 3) evidence-based practice; 4) quality improvement; 5) safety; and 6) informatics. A framework should minimally include, for each competency, a precise definition, the knowledge/skills/attitudes to be acquired, the expected levels of development, and indicators to assess the learner's progress (Poumay \& Georges, 2017).

The expected levels of development serve to illustrate each goal to be achieved while developing a competency. The way competencies are assessed must be consistent with these goals, i.e., holistic and not focused solely on technical skills. While the development and implantation of a competency framework is common in pre-licensure programs, few health care organizations have adopted this approach to support the professional development of nurses. In a 3-year pre-licensure program, a framework could describe expected levels at the completion of the first, second and third year, for each competency. In clinical settings, a similar framework could be designed, with expected levels after one, three and five years of professional practice.

Studies show that the competencies of NGNs improve rapidly during the first year of practice (Ke, Kuo, \& Hung, 2017; Lima, Newall, Jordan, Hamilton, \& Kinney, 2016). However, without a framework to define the competencies to be developed and the expected levels, nurse managers and clinical educators express different expectations (Freeling \& Parker, 2015; Numminen et al., 2014), which can be confusing for NGNs. Blanchet Garneau et al. (2017) point out that this confusion may also stem from individual interpretations of the intentions behind an expected level of competency: some interpret it as a safe minimum level of care and others see it as a goal for excellence. A well-structured competency framework could provide a clear trajectory for all nurses, including tools to assess competencies and orient their professional development.

\section{Contextualized Practice}

A competency is always oriented towards a goal and an action (in the broad sense of the term), articulated within a specific context. In nursing, the goal is always to provide a quality of care. The contexts in which students and nurses are immersed includes not only the physical environment around them but also the social, cultural and political environment. 
This context is dynamic; it evolves with the people of whom it is composed, and it adapts to changes in policies. By working in a clinical setting, nurses influence the context, which in turn will also influence the behavior of nurses.

Researchers (Numminen, Leino-Kilpi, Isoaho, \& Meretoja, 2015) have identified organizational factors that have a statistically significant influence on the competencies of nurses (ethical climate and practice environment), in addition to individual factors (empowerment and professional commitment). Charette et al. (2019b) also identified organizational and individual factors, different from those of Numminen et al. (2015). These organizational factors are the characteristics of the orientation period, the stability on a unit of care and a shift, the workload and the scientific culture of the care settings, whereas the individual factors were the personality and the previous experience of newly graduated nurse placements, support or intimidation by other nurses, and recognition of nursing expertise by other health professionals. Since these factors influence the practice context and the deployment of nurses' competencies, academics and clinical educators should not ignore them, but rather take them into account when designing learning activities. In this way, one promotes the application and transfer of knowledge to this real, imperfect and dynamic context.

\section{Optimal Quality of Care}

The deployment of competencies in a clinical setting aims to provide quality care to the population. The concept of quality of care is dynamic and made up of several dimensions: effectiveness, efficiency, accessibility, acceptability (or patient-centered care), equity, and safety (World Health Organization, 2006). Beyond the central concepts related to the development and deployment of competencies, the model presented in this paper emphasizes the continuity and interrelationship between nursing education and practice towards this goal of optimal quality of care. It could therefore be used by academics and clinical educators as well as having implications for research which will be discussed in the following section.

\section{Discussion}

This article presents a new model which calls on nurse academics and clinical educators to develop a common understanding of the interrelationship between the development of nursing competencies and their deployment in nursing practice. Although it has not yet been empirically validated, a first qualitative study was conducted using this model as a framework (Charette et al., 2019a, 2019b). This study corroborated the model's structure and identified factors influencing deployment of competencies.

\section{Implications for Research}

Empirical results are needed in order to understand how CBE programs prepare graduate nurses for independent practice in our complex interprofessional health care systems. Yet, little empirical knowledge is available on the impact of reformed CBE programs, including outcomes on the competencies implemented by nurses graduating from these programs (Morcke, Dornan, \& Eika, 2013). National nursing regulatory associations have advocated for the development of nursing education research. The Canadian Association of Schools of Nursing advocates for the development of nursing education research to promote disciplinary knowledge and contribution to quality health care (Canadian Association of Schools of Nursing, 2014). In the United States, the National League for Nursing supports the development of research methods to measure program outcomes and links to patient care (National League for Nursing, 2016). Curriculum reform in nursing has been one response from some academic institutions to recommendations of national and international organizations to improve the quality of care. However, these innovative 
programs require significant adaptation efforts by students and academics, not to mention the mobilization of human and financial resources (Goudreau et al., 2009).

This model of competency development and deployment in nursing practice could be used as a framework by researchers to study the impacts of CBE programs on NGNs' competencies or the influence of contexts on nursing practice. Neglecting to consider the influence of a context on the action of the professional seems incompatible with the holistic focus of the discipline, in addition to being inconsistent with the holistic perspective of a competency.

Finally, this model invites researchers to think about the connection between nurses' education and the deployment of their competencies, a relationship rarely examined in the literature.

\section{Implications for Pre-licensure and Continuing Education}

By integrating concepts from educational sciences and nursing, this model of competency development and deployment in nursing practice promotes the development of active TLMs based on authentic situations. More specifically, academics working in universities could use this model to gain a better understanding of how to prepare students to act in a professional context. Since a competency is a symbiosis of knowledge, skills and attitude in action (Charette et al., 2014), it is not enough to transmit theoretical or technical knowledge; students need to be able to act (and react) in an authentic and complex situation. The presented model considers various factors influencing the deployment of competencies in order to better prepare students for the reality of clinical settings.

This model also emphasizes the need to develop and use a competency framework to guide nursing programs. Such frameworks should, at the very least, describe the level of competency that should be attained by the completion of each year of a program. A competency framework also promotes coherence from one course to another throughout the program and gives clear indications of expectations. Although each academic institution develops its own framework, the competencies are often similar and consistent with the Quality and Safety Education for Nurses framework (Cronenwett et al., 2007). In clinical settings, consideration needs to be given to adopting a framework, since several options are possible. If an organization has a partnership with an academic institution, the simplest option is to use the same framework by adding expected levels of competency development after graduation. Another option could be to develop a framework based on an existing one, adapting it to the specificities of the clinical setting.

Such a framework could guide transition-to-practice programs or continuing education, by identifying the competencies to be deployed by all practicing nurses and the expected levels of competency. These expected levels should be identified empirically, through the construction of a cognitive learning model for each competency (Tardif, 2006). These models identify critical learning to be achieved by the learner in order to evolve to a new stage of expertise, which represents a cognitive reorganization process (Tardif, 2017).

For clinical educators, this model of nursing practice development and deployment highlights the interdependence of theory and practice, as well as the importance of using active TLMs to support competency development. Clinical educators could also use such a model to consider organizational and individual factors in developing transition and orientation programs for NGNs or to develop tools to facilitate the monitoring of competency development and deployment. 


\section{Limitations}

The major limitation of this model is that it is the result of a theoretical integration. Futures studies will be necessary to validate it.

\section{Conclusion}

The model for competency development and deployment in nursing practice that has been presented in this article integrates two commonly-cited approaches used in nursing education: second-generation CBE, from educational sciences, and Benner's work on the development of professional expertise. Further studies will be needed to enhance or empirically validate this model. However, the model contributes to creating a common language for nurse academics and clinical educators to narrow the gap between theory and practice, a gap that presently leads to a difficult transition for NGNs entering clinical settings. This model suggests that competency development does not cease at graduation and that learning activities in both pre-licensure programs and continuing education should be derived from active TLMs and pedagogical principals, as well as reflecting an authentic context or situation of care. This facilitated dialogue is necessary to support the integration of NGNs at the beginning of their practice and aims to support the optimal deployment of their competencies to ensure quality care for the population. 


\section{References}

Benner, P. (1984). From novice to expert: excellence and power in clinical nursing practice. Menlo Park, CA: Addison-Wesley Publishing.

Benner, P., Sutphen, M., Leonard, V., \& Day, L. (2010). Educating Nurses: A Call for Radical Transformation. San Francisco, CA: Jossey-Bass.

Benner, P., Tanner, C., \& Chesla, C. (1992). From beginner to expert: gaining a differentiated clinical world in critical care nursing. Advances in Nursing Science, 14(3), 13-28. https://doi.org/10.1097/00012272-199203000-00005

Benner, P., Tanner, C., \& Chesla, C. (2009). Expertise in Nursing Practice: Caring, Clinical Judgment, and Ethics (2 ed.). New York, NY: Springer Publishing Company.

Billings, D. M., \& Halstead, J. A. (2016). Teaching in Nursing: A Guide for Faculty (5 ed.). St-Louis, MO: Elsevier.

Blanchet Garneau, A., Lavoie, P., \& Grondin, M. (2017). Dichotomy and dialogue in conceptualizations of competency in health professionals' education. Journal of Nursing Education and Practice, 7(6), 18-26. https://doi.org/10.5430/jnep.v7n6p18

Boulet, A., Savoie-Zajc, L., \& Chevrier, J. (1996). Les stratégies d'apprentissages à l'université. Québec, QC: Presses de l'Université du Québec.

Canadian Association of Schools of Nursing. (2014). Strategic Plan 2014 - 2018: Driving nursing education that transforms healthcare. Retrieved from http://casn.ca//wpcontent/uploads/2014/12/StratplanBillingual.pdf

Charette, M., Goudreau, J., \& Alderson, M. (2014). Une analyse évolutionniste du concept de compétence. Recherche En Soins Infirmiers(116), 27-39. https://doi.org/10.3917/rsi.116.0028

Charette, M., Goudreau, J., \& Bourbonnais, A. (2019a). How do new graduate nurses from a competency-based program demonstrate their competencies? A focused ethnography of acute care settings. Nurse Education Today, 79, 161-167. https://doi.org/10.1016/j.nedt.2019.05.031

Charette, M., Goudreau, J., \& Bourbonnais, A. (2019b). Organizational and individual factors influencing the practice of new graduate nurses: a focused ethnography of acute care settings. Journal of Clinical Nursing, 28(19/20), 3618-3631.

https://doi.org/10.1111/jocn.14959

Cronenwett, L., Sherwood, G., Barnsteiner, J., Disch, J., Johnson, J., Mitchell, P., . . . Warren, J. (2007). Quality and safety education for nurses. Nursing Outlook, 55(3), 122-131. https://doi.org/10.1016/j.outlook.2007.02.006

Dadgaran, I., Parvizy, S., \& Peyrovi, H. (2012). A global issue in nursing students' clinical learning: the theory-practice gap. Procedia-Social and Behavioral Sciences, 47, 1713-1718. https://doi.org/10.1016/j.sbspro.2012.06.888

Dewey, J. (1997). Experience and education. New York, NY: Simon \& Schuster.

Dreyfus, S. E., \& Dreyfus, H. L. (1980). A five-stage model of the mental activities involved in directed skill acquisition. Berkeley, CA: University of California.

Duchscher, J. B. (2009). Transition shock: the initial stage of role adaptation for newly graduated Registered Nurses. Journal of Advanced Nursing, 65(5), 1103-1113. https://doi.org/10.1111/j.1365-2648.2008.04898.x 
Flexner, A. (1910). Medical education in the United Stated and Canada. Retrieved from http://www.carnegiefoundation.org/sites/default/files/elibrary/Carnegie_Flexner_Rep ort.pdf

Franklin, N., \& Melville, P. (2015). Competency assessment tools: An exploration of the pedagogical issues facing competency assessment for nurses in the clinical environment. Collegian, 22(1), 25-31. https://doi.org/10.1016/j.colegn.2013.10.005

Freeling, M., \& Parker, S. (2015). Exploring experienced nurses' attitudes, views and expectations of new graduate nurses: A critical review. Nurse Education Today, 35(2), e42-e49. https://doi.org/10.1016/j.nedt.2014.11.011

Frenk, J., Chen, L., Bhutta, Z. A., Cohen, J., Crisp, N., Evans, T., . . Zurayk, H. (2010). Health professionals for a new century: transforming education to strengthen health systems in an interdependent world. The Lancet, 376(9756), 1923-1958. https://doi.org/10.1016/s0140-6736(10)61854-5

Garside, J. R., \& Nhemachena, J. Z. Z. (2013). A concept analysis of competence and its transition in nursing. Nurse Education Today, 33(5), 541-545. https://doi.org/10.1016/j.nedt.2011.12.007

Gonczi, A. (1996). Reconceptualising competency-based education and training: with particular reference to education for occupations in Australia. (Doctoral dissertation), University of Technology, Sydney.

Gonczi, A., \& Hager, P. (2010). The competency model. In P. Peterson, E. Baker, \& B. McGaw (Eds.), International Encyclopedia of Education (Vol. 8, pp. 403-410). Oxford, United Kingdom: Elsevier.

Goudreau, J., Pepin, J., Dubois, S., Boyer, L., Larue, C., \& Legault, A. (2009). A second generation of the competency-based approach to nursing education. International Journal of Nursing Education Scholarship, 6(1), Article 15. https://doi.org/10.2202/1548-923x.1685

Guillemette, F., \& Gauthier, C. (2008). L'approche par compétences (APC) en formation des maîtres: Analyse documentaire et critique. Recherches \& éducations. Retrieved from http://journals.openedition.org/rechercheseducations/84

Hatlevik, I. K. R. (2012). The theory-practice relationship: reflective skills and theoretical knowledge as key factors in bridging the gap between theory and practice in initial nursing education. Journal of Advanced Nursing, 68(4), 868-877. https://doi.org/10.1111/j.1365-2648.2011.05789.x

Institute of Medicine. (2003). Health Professions Education: A Bridge to Quality. Washington, D.C.: The National Academies Press.

Ishler, R. E. (1974). Competency-based teacher education: A potpourri of perspectives. Washington, D.C.: Association of Teacher Educators.

Ke, Y. T., Kuo, C. C., \& Hung, C. H. (2017). The effects of nursing preceptorship on new nurses' competence, professional socialization, job satisfaction and retention: A systematic review. Journal of Advanced Nursing, 73(10), 2296-2305. https://doi.org/10.1111/jan.13317

Lasnier, F. (2000). Réussir la formation par compétences. Montréal, QC: Guérin. 
Lima, S., Newall, F., Jordan, H. L., Hamilton, B., \& Kinney, S. (2016). Development of competence in the first year of graduate nursing practice: a longitudinal study. Journal of Advanced Nursing, 72(4), 878-888. https://doi.org/10.1111/jan.12874

Meretoja, R., \& Koponen, L. (2012). A systematic model to compare nurses' optimal and actual competencies in the clinical setting. Journal of Advanced Nursing, 68(2), 414422. https://doi.org/10.1111/j.1365-2648.2011.05754.X

Morcke, A. M., Dornan, T., \& Eika, B. (2013). Outcome (competency) based education: an exploration of its origins, theoretical basis, and empirical evidence. Advances in Health Sciences Education, 18, 851-863. https://doi.org/10.1007/s10459-012-9405-9

Morgan, D. L. (2014). Pragmatism as a Paradigm for Social Research. Qualitative Inquiry, 20(8), 1045-1053. https://doi.org/10.1177/1077800413513733

National League for Nursing. (2016). NLN Research Priorities in Nursing Education 20162019. Retrieved from http://www.nln.org/docs/default-source/professionaldevelopment-programs/nln-research-priorities-in-nursing-education-singlepages.pdf?sfvrsn=2

Numminen, O., Laine, T., Isoaho, H., Hupli, M., Leino-Kilpi, H., \& Meretoja, R. (2014). Do educational outcomes correspond with the requirements of nursing practice: educators' and managers' assessments of novice nurses' professional competence. Scandinavian Journal of Caring Sciences, 28, 812-821. https://doi.org/10.1111/scs.12115

Numminen, O., Leino-Kilpi, H., Isoaho, H., \& Meretoja, R. (2015). Newly Graduated Nurses' Competence and Individual and Organizational Factors: A Multivariate Analysis. Journal of Nursing Scholarship, 1-12. https://doi.org/10.1111/jnu.12153

Pastré, P. (1999). La conceptualisation dans l'action: bilan et nouvelles perspectives. Éducation permanente, 139, 13-35.

Perrenoud, P. (2004). Adosser la pratique réflexive aux sciences sociales, condition de la professionnalisation. In J.-F. Inisan (Ed.), Analyse de pratiques et attitude réflexive en formation (pp. 11-32). Reims, France: CRDP de Champagne-Ardenne.

Poumay, M., \& Georges, F. (2017). Des balises méthodologiques pour construire un référentiel de compétences et une grille de programme. In M. Poumay, J. Tardif, \& F. Georges (Eds.), Organiser la formation à partir des compétences : Un pari gagnant pour l'apprentissage dans le supérieur. Louvain-la-Neuve, Belgique: De Boeck Supérieur.

Rischel, V., Larsen, K., \& Jackson, K. (2008). Embodied dispositions or experience? Identifying new patterns of professional competence. Journal of Advanced Nursing, 61(5), 512-521. https://doi.org/10.1111/j.1365-2648.2007.04543.x

Risjord, M. (2010). Nursing Knowledge. Science, Practice and Philosophy. Chichester, United Kingdom: Wiley-Blackwell.

Saifan, A., AbuRuz, M. E., \& Masa'deh, R. (2015). Theory Practice Gaps in Nursing Education: A Qualitative Perspective. Journal of Social Sciences/Sosyal Bilimler Dergisi, 11(1). https://doi.org/10.3844/jssp.2015.20.29

Schön, D. A. (1984). The Reflective Practitioner: How Professionals Think In Action. New York, NY: Basic Books. 
Shabani, K., Khatib, M., \& Ebadi, S. (2010). Vygotsky's Zone of Proximal Development: Instructional Implications and Teachers' Professional Development. English Language Teaching, 3(4), 237-248. https://doi.org/10.5539/elt.v3n4p237

Smith, S. A. (2012). Nurse Competence: A Concept Analysis. International Journal of Nursing Knowledge, 23(3), 172-182. https://doi.org/10.1111/j.2047$\underline{3095.2012 .01225 . \mathrm{X}}$

Steiner, G. (2001). Transfer of Learning, Cognitive Psychology of. In N. J. Smelser \& P. B. Baltes (Eds.), International Encyclopedia of the Social \& Behavioral Sciences (2 ed., pp. 15845-15851). Oxford, United Kingdom: Pergamon.

Tardif, J. (2006). L'évaluation des compétences - Documenter le parcours de formation. Montréal, QC: Chenelière Éducation.

Tardif, J. (2016). Ancrer les apprentissages sur les connaissances antérieures des étudiants. In T. Pellascia (Ed.), Comment (mieux) évaluer et former les étudiants en médecine et en sciences de la santé?. Louvain-la-neuve, Belgique: deBoeck Supérieur.

Tardif, J. (2017). Des repères conceptuels à propos de la notion de compétence, de son développement et de son évaluation. In M. Poumay, J. Tardif, \& F. Georges (Eds.), Organiser la formation à partir des compétences : Un pari gagnant pour l'apprentissage dans le supérieur. Louvain-la-Neuve, Belgique: De Boeck Supérieur.

Weir, G. M. (1932). Survey of Nursing Education in Canada. Vancouver, BC: University of British Columbia.

World Health Organization. (2006). Quality of Care: A Process for making Strategic Choices in Health Systems. Retrieved from http://www.who.int/management/quality/assurance/QualityCare_B.Def.pdf 